\title{
A numerical study of Richtmyer-Meshkov instability in continuously stratified fluids
}

\author{
T. Pham a) and D. I. Meiron \\ Applied Mathematics 217-50, 319 Firestone Laboratory, California Institute of Technology, Pasadena, \\ California 91125
}

(Reccived 3 April 1992; acccpted 1 October 1992)

\begin{abstract}
Theory and calculations are presented for the evolution of Richtmyer-Meshkov instability in two-dimensional continuously stratified fluid layers. The initial acceleration and subsequent instability of the fluid layer are induced by means of an impulsive pressure distribution. The subsequent dynamics of the fluid layer are then calculated numerically using the incompressible equations of motion. Initial conditions representing single-scale perturbations and multiple-scale random perturbations are considered. It is found that the growth rates for Richtmyer-Meshkov instability of stratified fluid layers are substantially lower than those predicted by Richtmyer for a sharp fluid interface with an equivalent jump in density. A frozen field approximation for the early-time dynamics of the instability is proposed, and shown to approximate the initial behavior of the layer over a time equivalent to the traversal of several layer thicknesses. It is observed that the nonlinear development of the instability results in the formation of plumes of penetrating fluid.

Late in the process, the initial momentum deposited by the impulse is primarily used in the internal mixing of the layer rather than in the overall growth of the stratified layer. At intermediate times, some evidence for the existence of scaling behavior in the width of the mixing layer of the instability is observed for the multiple-scale random perturbations, but not for the single-scale perturbations. The time variation of the layer thickness differs from the scaling derived using ideas of self-similarity due to Barenblatt [Non-Linear Dynamics and Turbulence, edited by G. I. Barenblatt, G. Ioos, and D. D. Joseph (Pitman, Boston, 1983), p. 48] even at low Atwood ratio, presumably because of the inhomogeneity and anisotropy due to the excitation of vortical plumes.
\end{abstract}

\section{INTRODUCTION}

It is well known that in a gravitational field directed downwards, a surface separating two fluids of different densities is stable provided the heavy fluid is at the bottom, and the lighter fluid is at the top. Any ripple on the surface due to perturbations will decay in the presence of viscosity and the surface returns to its normal flat shape. On the other hand, if we accelerate the whole system with a downward acceleration of magnitude greater than that of the gravitational field, any ripple on the surface will grow exponentially fast. Late in the process, spikes of heavy fluid penetrate the lighter fluid. This is an example of the wellknown Rayleigh-Taylor instability. This instability arises from the misalignment between the pressure gradient and the density gradient, which is responsible for the generation of the vorticity in the flow. Another instability caused by this misalignment is the Richtmyer-Meshkov instability due to the passage of a shock wave normal to a perturbed interface.

If the compressibility of the fluids is neglected, the Richtmyer-Meshkov instability can be considered as the limit of the Rayleigh-Taylor instability when the acceleration is impulsive, i.e., it acts for almost zero time but the

\footnotetext{
a) Present address: Department of Mechanical Engineering, Massachusetts Institute of Technology, 77 Massachusetts Ave., Cambridge, Massachusetts 02139 .
}

magnitude of the acceleration is very large so that a finite amount of momentum is transferred to the fluid as in the case of a shock. This approximation is reasonable when the shock strength is weak. Its validity has been verified by Richtmyer ${ }^{l}$ in his consideration of the effect of compressibility on the initial growth rate of a perturbed interface. $\mathrm{He}$ concluded that for a weak shock, the extension of the Rayleigh-Taylor formula for a constant acceleration of finite magnitude to an impulsive one yields results which are accurate to within $5 \%-10 \%$ of the results obtained from a full compressible calculation. It is important to note that a fundamental difference between the two instabilities, Rayleigh-Taylor and Richtmyer-Meshkov, is that in the absence of gravity, the Rayleigh-Taylor instability occurs only when a light fluid is accelerated into a heavy one but not in the opposite direction, while there is no directional dependence for Richtmyer-Meshkov instability. Note also, that the growth of the Richtmyer-Meshkov is initially linear in time, in contrast to the exponential growth exhibited by the Rayleigh-Taylor instability.

Saffman and Meiron ${ }^{2}$ used this impulse approximation in a slightly different way for a continuously stratified fluid, and showed that the growth of the instability is reduced when one decreases the density gradient across the interface. Their conclusion was based on calculations of the kinetic energy imparted to the fluid by an impulse. Studies of the effect of smoothing the density gradient on the growth rate of Rayleigh-Taylor instability have also been 
carried out by LeLevier et al., ${ }^{3}$ Duff et al., ${ }^{4}$ and others. Mikaelian ${ }^{5}$ has studied the effect of stratification on Richtmyer-Meshkov growth rates by approximating the continuous density gradient as a sequence of discrete density jumps. In further work ${ }^{6}$ he applies this technique to show that density gradient stabilization is more effective for Richtmyer-Meshkov instability than for RayleighTaylor instability. He has also made use of the relationship between kinetic energy and growth rate as a means of comparing Richtmyer-Meshkov growth rates with those of the better known Rayleigh-Taylor instability. ${ }^{7}$

Meshkov ${ }^{8}$ experimentally observed the linear growth rate predicted from Richtmyer's theory, and also noted the directional independence of this instability. Richtmyer's original theoretical work had only considered shock propagation from light to heavy media. Recently, Brouillette ${ }^{9}$ has investigated the shock-induced Richtmyer-Meshkov instability of a sharp and smoothed density gradient, and verified the reduction of the growth rate due to a smooth density gradient. Mikaelian ${ }^{6}$ has shown that the reduction in growth rate observed experimentally is consistent with the theoretical estimates discussed above.

'Ihe theories of Richtmyer, Mikaelian, and of Saffman and Meiron are applicable for small perturbations on the interface. In the present investigation, we examine both small- and finite-amplitude perturbations of the interface. We perform long-time numerical simulations of the instability. We consider the effect of the density gradient on the growth rate, the detailed structure of the time evolution of a single-scale perturbation, and of a randomly perturbed interface.

We also attempt to address the question of the existence of a self-similar long-time asymptotic limit of the Richtmyer-Meshkov instability, which is known to exist for the Rayleigh-Taylor instability. From dimensional analysis of the dominant length scale in the late stage of the Rayleigh-Taylor instability (when memory of the initial configuration has been lost), it can be shown that the width of the mixed region is proportional to $g t^{2}$, where $g$ is the effective acceleration, and $t$ is time. Youngs ${ }^{10}$ has performed extensive numerical simulations of twodimensional (2-D) Rayleigh-Taylor instability. In examining the penetration of the heavy fluid by the light fluid, he finds that this scaling behavior is verified provided that the initial perturbation is random and uniformly distributed. In other words, the quadratic law ceases to be valid if large-amplitude long wavelength perturbations are initially present. This result is also confirmed experimentally by Read. ${ }^{11}$

In contrast to the Rayleigh-Taylor instability in which there is a continuous flow of energy into the system, i.e., constant acceleration of finite amplitude, in RichtmyerMeshkov instability the momentum is only deposited at the interface initially by the traversal of the shock. Moreover, as noted by Brouillette, for a single-scale perturbation, the deposited energy is a function of the initial wavelength for Richtmyer-Meshkov instability, but not for the RayleighTaylor instability. From these considerations, Brouillette conjectures that a power law governing the mixing of
Richtmyer-Meshkov instability can hardly exist at late times. In experiments performed by Brouillette, ${ }^{9}$ it was found that perturbations of an interface evolve quickly into the nonlinear turbulent mixing regime, while those of a continuous density gradient exhibit growth only at late times. For both cases, scaling behavior was not observed.

On the other hand, from a consideration of the time evolution of initial turbulent energy deposited at a plane interface, there seems to be another possibility as indicated by the work of Barenblatt. ${ }^{12}$ Using a turbulent energy balance equation whose closure is accomplished by certain Kolmogorov-type similarity hypotheses, together with an assumption that the turbulence length scale is equal to a certain fixed part of actual turbulent layer depth, Barenblatt is able to show that there exists a self-similar asymptotic solution for the propagation of turbulence from an instantaneous plane source originally concentrated in a horizontal plane layer of finite depth.

For the idealized problem of the motion of an incompressible inviscid homogeneous fluid (corresponding in this case to density ratio unity or Atwood number zero) driven by an infinitely thin instantaneous turbulence source of finite bulk intensity, Barenblatt shows that the depth of the turbulent layer grows as $t^{2 / 3}$ from a dimensional argument. For a viscous fluid, he finds that a self-similar solution can only be obtained if the initial depth of the turbulent layer is different from zero. The self-similarity, however, is incomplete, i.e., it only exists for intermediate time, and is of the second kind so that the time exponent in self-similar variables cannot be determined from purely dimensional considerations. The value of this exponent is instead obtained from a nonlinear eigenvalue problem, and shown to range from 0 to $2 / 3$. As mentioned above, this result is thought to apply to the late-time growth of Richtmyer-Meshkov instability at low-density contrast but it has never been verified.

Results of numerical simulations presented here seem to indicate the existence of scaling behavior at intermediate time for the width of the stratified layer having initial multiple-scale perturbations. The scaling shows a weak dependency on the initial configurations with the time exponent lying in the neighborhood of $1 / 4$. On the other hand, results from single-scale perturbations definitely show a lack of universality relative to the initial conditions. For single-scale perturbations, this result appears to verify the observation of Brouillette that the details of the instability depend strongly on the initial wavelength. Our results on the existence of scaling behavior should be viewed as preliminary. Due to limits on our present computational resources, a much longer simulation with higher resolution is not possible. The discrepancy of the behavior exhibited by our simulations and the predictions of Barenblatt (even at low Atwood ratio) is presumably due to the existence of inhomogeneity and anisotropy arising from the excitation of vortical plumes as opposed to the uniform character of the random turbulence assumed in the work of Barenblatt.

In Sec. II we review the impulse approximation made by Saffman and Meiron and others which models the effect of the interaction of a weak shock with a stratified layer of 
fluid as a pressure impulse. That this can be an adequate approximation is discussed in the thesis of Pham. ${ }^{13}$ Pham considers the effect of a normal shock incident on both a discretely stratified fluid and a continuously stratified fluid. The effect of a shock on a discrete interface is studied using standard gas dynamics; the effect of a shock on a continuously stratified fluid is studied using Chester-Chisnell theory. In both cases Pham neglects perturbations on the interface concentrating instead on the basic motion initiated by the shock. This gives some feeling for the range of shock Mach numbers over which alteration of the initial density gradient by the shock (due to compression) is an important effect. It is found that the impulsive approximation is valid for weak shocks. With this result in mind, we then formulate the equations of motion for the subsequent motion of the layer once the shock has passed.

In Sec. III we briefly describe the numerical techniques used to simulate the equations of motion and to set up the appropriate impulse initial conditions. Details of the numerical method are relegated to the Appendix. Our results are described in Sec. IV followed by conclusions in Sec. V.

\section{IMPULSE APPROXIMATION}

In this study, we hope to gain some insight into the long-time behavior as well as the effect of smoothness of the density gradient on the growth rate of the instability using a full 2-D numerical simulation of the timedependent incompressible equations. We model the initial effect of compressibility by approximating the action of the shock as an impulsive pressure using an incompressible impulsive theory. This idea was used by Saffman and Meiron $^{2}$ to study the kinetic energy deposited by an impulse at a layer of stratified fluid with a weak density perturbation in the direction transverse to the flow. This incompressible approximation is a reasonable one for an impulsive acceleration created by a weak shock, since the induced velocity is subsonic. Moreover, one expects that for such weak shocks, the effect of compressibility is limited to some initial modification of the density distribution, since the residence time of the shock in the nonuniform region is very small compared to the characteristic time for the evolution of the instability. Some justification for the use of the incompressible impulsive approximation as well as an assessment of its range of validity is provided by examining the solution of a planar shock passing a region of nonuniform density distribution using one-dimensional gas dynamics. Details of this study are presented in the thesis of Pham. ${ }^{13}$ In this section, we will only summarize the time-dependent equations governing the instability.

Saffman and Meiron modeled the motion of a perturbed planar interface undergoing a shock-induced acceleration as one generated by the impulsive motion of the containing walls with a velocity $V$ directed parallel to the undisturbed density gradient. Since the fluid is incompressible, this motion induces instantaneously a pressure field $P(x, y) \delta(t)$, where $\delta(t)$ is the delta function, and there exists a balance between the pressure gradient and the acceleration (viscous and inertial terms are negligible). Note that the function $P(x, y)$ has units of pressure timcs time.
Let $y$ denote the vertical coordinate parallel to the undisturbed density gradient, and $x$ be the horizontal coordinate. The upper fluid has density $\rho_{+}$, and the lower fluid has density $\rho_{-}$as $y \rightarrow \pm \infty$, respectively. Integrating the momentum equations, one obtains the equations for the induced initial velocities,

$$
\begin{aligned}
& u=-\frac{1}{\rho_{0}} \frac{\partial P}{\partial x} H(t), \\
& v=-\frac{1}{\rho_{0}} \frac{\partial P}{\partial y} H(t),
\end{aligned}
$$

where $H(t)$ is the Heaviside function, and $\rho_{0}$ is the density distribution at the time $t=0^{-}$. Using the continuity equation, the impulsive pressure is determined by

$$
\frac{\partial}{\partial x}\left(\frac{1}{\rho_{0}} \frac{\partial P}{\partial x}\right)+\frac{\partial}{\partial y}\left(\frac{1}{\rho_{0}} \frac{\partial P}{\partial y}\right)=0 .
$$

The boundary conditions are

$$
\begin{aligned}
& \frac{\partial P}{\partial y} \rightarrow-\rho_{+} V \text { as } y \rightarrow+\infty, \\
& \frac{\partial P}{\partial y} \rightarrow-\rho_{-} V \text { as } y \rightarrow-\infty .
\end{aligned}
$$

At a vertical wall $x=$ const, there is no flux. Hence $\partial P / \partial x$ $=0$. From the divergence theorem, it can be shown that the solution of the Poisson equation (3) is unique up to an arbitrary constant if the boundary conditions satisfy the compatibility condition,

$$
\int_{\Omega} \nabla \cdot\left(\frac{1}{\rho} \nabla p\right) d \Omega=\int_{S} \frac{1}{\rho} \nabla p \cdot \mathbf{n} d S .
$$

The boundary conditions (4) meet this requirement, and hence the solution for the initial velocity of the impulsive problem is well posed. Saffman and Meiron solved this system of equations for a density profile of the form

$$
\rho_{0}=\bar{\rho}(y)+\epsilon \rho^{\prime}(x, y),
$$

where $\epsilon$ is a small perturbation parameter. In particular, they chose the following density profile,

$$
\rho_{0}=1+A \tanh \left(\frac{y}{L}\right)+\left(\frac{\epsilon}{2 L}\right) \operatorname{sech}^{2}\left(\frac{y}{L}\right) \cos (k x),
$$

where $L$ is the characteristic width of the stratified layer. This density profile includes the first two terms of the Taylor expansion of a more general profile,

$$
\rho_{0}=1+A \tanh \left(\frac{1}{L}\left[y-\epsilon^{\prime} \cos (k x)\right]\right),
$$

where $\epsilon^{\prime}$ is related to $\epsilon$ by

$$
c^{\prime}=-\epsilon / 2 A \text {. }
$$

With this distribution, they were able to analyze the problem using perturbation theory. The dependency of $\rho^{\prime}$ on $x$ is essential, since there is no generation of vorticity unless there is a density perturbation in the $x$ direction. 
For small disturbances, Saffman and Meiron predict that less kinetic energy is generated when the width of the undisturbed density distribution is increased. Hence, in order to reduce the Richtmyer-Meshkov instability, one should decrease the density gradient along the direction of the shock.

In this study, the solution of the incompressible impulsive model is used as the initial condition for a fully nonlinear simulation of the finite-amplitude stage of the instability. The subsequent flow is governed by the twodimensional unsteady Navier-Stokes equations,

$$
\begin{aligned}
& \frac{\partial \rho}{\partial t}+\mathbf{u} \cdot \nabla \rho=0, \\
& \rho \frac{\partial \mathbf{u}}{\partial t}+\rho \mathbf{u} \cdot \nabla \mathbf{u}=-\nabla P+\mu \nabla^{2} \mathbf{u}, \\
& \nabla \cdot \mathbf{u}=0,
\end{aligned}
$$

where $\mu$ is the viscosity of the fluid. The above equations are solved subject to the boundary conditions

$$
\begin{aligned}
& \mathbf{u} \rightarrow(0, V) \text { as } \boldsymbol{y} \rightarrow \pm \infty, \\
& \rho \rightarrow \rho_{ \pm} \text {as } y \rightarrow \pm \infty .
\end{aligned}
$$

The velocity $\mathbf{u}$ and density $\rho$ are periodic in $x$, with period $2 \pi / k$. Since we are interested in the inviscid regime, the magnitude of the viscosity used is very small. It is present only to stabilize the smallest and ill-resolved scales of the numerical scheme. We scale the above equations using the following characteristic quantities:

$$
\begin{aligned}
& u_{c}=|V|, \\
& l_{c}=L, \\
& p_{c}=\rho_{c} u_{c}^{2}, \\
& \rho_{c}=\rho_{+}+\rho_{-}, \\
& t_{c}=L / u_{c} .
\end{aligned}
$$

In both cases, the equations have the same forms as shown in Eqs. (10)-(12), except that in the nondimensional momentum equation the viscosity $\mu$ in Eq. (11) is replaced by the factor $\mathrm{Re}^{-1}$ where

$$
\operatorname{Re}=\rho_{c} u_{c} l_{d} / \mu
$$

is the Reynolds number. In the actual simulations, we choose $V=-1, k=1$, and $\mu=10^{-6}$.

\section{NUMERICAL METHOD FOR SIMULATION}

Due to the sharp gradient of the initial density profile in some parts of the flow domain, we employ a nonuniform grid in a moving frame in order to resolve this region. We use a finite-difference discretization with a staggered grid and with the primitive variables as the unknowns. The system of equations is discretized in time with an implicit Crank-Nicolson scheme applied to all terms. Central differencing is used to compute spatial derivatives. The result- ing nonlinear set of equations is solved by an iterative technique usually known as the artificial compressibility method. ${ }^{14,15}$

Following Saffman and Meiron, we consider a particular form of the initial density as given in Eq. (8). This density. profile has a small variation in the transverse $x$ direction while it changes rapidly about the origin along the flow $(y)$ direction. Hence in the $y$ direction, we use the following coordinate transformation:

$$
\eta=\tanh (\gamma y),
$$

where $\gamma$ is a controlling parameter for stretching.

A uniform distribution of grid points in the $\eta$ computational domain corresponds to a nonuniform distribution over an increasing range in $y$ in the physical domain. As $\gamma \rightarrow 0$, we have a linear transformation and the discretization in $y$ becomes uniform. As $\gamma \rightarrow \infty$, we have a very dense distribution of grid points around $y=0$. This transformation is convenient because of the monotonicity and the smoothness of the hyperbolic tangent. The transformed equations are described in the Appendix.

In all of the calculations, we use a staggered grid in the $x, \eta$ domain. The flow region is divided up three different ways resulting in three kinds of computational cells: the $u$, $v$, and $p$ cell. For the $u$ cell, the center of the cell is the node point for the $u$ component of the velocity. The density and the pressure equation are discretized at the center of the $p$ cell. The $u$-momentum equation and the $v$-momentum equation are discretized at the center of the $u$ and $v$ cells, respectively.

The initial velocity ficld is obtained by first solving Eq. (3) and the corresponding boundary conditions (4) for the impulsive pressure $P(x, y)$. Note that $P(x, y)$ actually has the units of pressure times time. It only has physical meaning when used along with the Heaviside function for computing the initial velocity field. In transformed coordinates these equations are

$$
\frac{\partial}{\partial x}\left(\frac{1}{\rho_{0}} \frac{\partial P}{\partial x}\right)+\frac{d \eta}{d y} \frac{\partial}{\partial \eta}\left(\frac{1}{\rho_{0}} \frac{d \eta}{d y} \frac{\partial P}{\partial \eta}\right)=0,
$$

and

$$
\frac{d \eta}{d y} \frac{\partial P}{\partial \eta}=-\rho_{ \pm} V \quad \text { as } \eta \rightarrow \pm 1
$$

The solution procedure is described in detail in the Appendix.

After solving for the impulsive pressure $P$, the initial velocity field is obtained by using Eqs. (1) and (2). The initial pressure is then determined automatically by the momentum equations. Before discussing the numerical solution for this initial pressure, however, we would like to formulate the problem in a reference frame moving with the fluid flow.

In a fixed reference frame, as the flow evolves, the fine resolution of the region with a sharp density gradient along the flow ( $y$ direction) will deteriorate in time. To avoid this difficulty, we solve the problem in a frame moving along with the flow in the negative $y$ direction with the far 
field velocity $V$. The governing equations in a general moving coordinate are given in Lamb. ${ }^{16}$ For our problem, the equations of motion become

$$
\begin{aligned}
& \frac{\partial u}{\partial t}+\frac{\partial}{\partial x}\left(u^{2}\right)+\frac{\partial}{\partial y}[u(v-V)]+\frac{1}{\rho} \frac{\partial p}{\partial x}-\frac{1}{\rho \operatorname{Re}} \nabla^{2} u=0, \\
& \frac{\partial v}{\partial t}+\frac{\partial}{\partial x}[u(v-V)]+\frac{\partial}{\partial y}\left[(v-V)^{2}\right]+\frac{1}{\rho} \frac{\partial p}{\partial y}-\frac{1}{\rho \operatorname{Re}} \nabla^{2} v \\
& =0 \text {, } \\
& \frac{\partial \rho}{\partial t}+u \frac{\partial \rho}{\partial x}+(v-V) \frac{\partial \rho}{\partial y}=0, \\
& \frac{\partial u}{\partial x}+\frac{\partial v}{\partial y}=0 \text {. }
\end{aligned}
$$

The boundary conditions for the velocity and the density in this moving frame remain the same as given in Eq. (13). In the remaining parts of this section, we will discuss the numerical solution for the initial pressure and subsequent motion.

The initial pressure is computed by using the method of Harlow and Welch. ${ }^{17}$ We first discretize the $u$, and $v$-momentum equation at the center of the $u$, and $v$ cell, respectively. The resulting equations are then used in the discretization of the equation,

$$
\boldsymbol{\nabla} \cdot \mathbf{u}_{t}=0,
$$

at the center of the $p$ cell. Imposing the continuity condition at time $t=\Delta t$, we obtain a discretized Poisson equation for the pressure. The solution of this equation and the velocity field generated from the impulsive acceleration provide the necessary initial conditions for a full simulation of the unsteady two-dimensional Navier-Stokes incompressible equations. We use an iterative scheme proposed by Soh and Goodrich ${ }^{14}$ with a modification to include the density equation. The overall iteration can be summarized as follows. First all terms of Eqs. (19)-(21) are discretized using the Crank-Nicolson scheme with central differencing for the space derivatives. Between two given physical time steps, this results in a nonlinear set of equations, which is formulated into a fictitious continuous "pseudotime" system, and is solved by the well-known artificial compressibility method. ${ }^{15}$ Details of the iteration procedure are provided in the Appendix.

\section{RESULTS}

The numerical results of the simulation of RichtmyerMeshkov instability are included in this section. We define average quantities to characterize the results of our numerical simulations and describe the two kinds of initial density distribution considered. Since the instability is weak, we also propose a model for its initial development based solely on the action of the vorticity field imposed by the impulsive acceleration. We call this the frozen field model, and include here a discussion on its formulation. We present the growth rates of a continuously stratified fluid layer as compared to those predicted by Richtmyer for a discretely stratified fluid interface with an equivalent jump in density. Finally, we only include some exemplary results of our simulations for both the single-scale and random multiple-scale perturbations of a stratified fluid. A more detailed description of the results can be found in the thesis of Pham. ${ }^{13}$

\section{A. Characteristic average quantities of the flow}

In order to characterize our results, we consider two kinds of average quantities for the density, representing averaging over the flow field in the $y$ direction and in the $x$ direction. The average over $y$ is a function of $x$ and is defined by

$$
\bar{\rho}_{y}(x)=\frac{2 Y \rho_{+}-\int_{-Y}^{Y} \rho(x, y) d y}{\rho_{+}-\rho_{-}}-Y,
$$

where $Y$ is the maximum value of $y$ in the region composed of the $v$ cells, and $\rho_{+}, \rho_{-}$is the uniform density obtained as $y \rightarrow \pm \infty$, respectively.

Equation (24) is a kind of area average of the density. The definition of $\bar{\rho}_{y}(x)$ allows us to specify an average interface location for the stratified layer. It gives the exact functional form of a sharp interface $y=f(x)$ which separates two regions of uniform density, $\rho_{+}$and $\rho_{-}$. Hence, this definition is convenient for comparison of the numerical results with the predictions of the Richtmyer theory, since an average amplitude $a(t)$, based on $\bar{\rho}_{y}(x)$, can be defined as

$$
a(t)=\frac{1}{2}\left\{\max \left[\bar{\rho}_{y}(x)\right]-\min \left[\bar{\rho}_{y}(x)\right]\right\} .
$$

The growth of the instability is characterized by the time derivative of $a$. We interpolate $\bar{\rho}_{y}(x)$ using cubic splines before $a$ is computed so that a smooth growth rate $d a / d t$ as a function of time is obtained.

The second density average is defined as follows:

$$
\bar{\rho}_{x}(y)=\frac{1}{2 \pi} \int_{0}^{2 \pi} \rho(x, y) d x
$$

which is the standard area average. From $\bar{\rho}_{x}(y)$, we can define a width $\delta$ of the stratified layer. Let $y_{+}$be the smallest value of $y$ such that

$$
\left|\bar{\rho}_{x}(y)-\rho_{+}\right| \leqslant d\left|\rho_{+}-\rho_{-}\right|,
$$

where $d$ is some specified cutoff. Similarly, let $y_{-}$be the largest value of $y$ such that

$$
\left|\bar{\rho}_{x}(y)-\rho_{-}\right| \leqslant d\left|\rho_{+}-\rho_{-}\right|
$$

Then, the width of the density layer is defined as

$$
\delta=y_{+}-y_{-} .
$$

In searching for $y_{+}$and $y_{-}$, we use linear interpolation with $d$ taken to be 0.02 , i.e., the width $\delta$ covers a distance over which the variation of the density within the layer is $98 \%$ of the maximum density jump. 
TABLE I. Ratio of the classical growth rate at a sharp interface [Eq. (39)] to the numerically calculated initial growth rate at a diffuse interface.

\begin{tabular}{|c|c|c|c|c|c|c|}
\hline$A$ & $\epsilon$ & $L=10.0$ & $L=1.0$ & $L=0.1$ & $L=0.01$ & $L=0.001$ \\
\hline \multirow[t]{4}{*}{ \pm 0.2} & 0.01 & 15.026 & 2.1280 & 1.1023 & 1.0283 & 1.0187 \\
\hline & 0.1 & 15.027 & 2.1304 & 1.1052 & 1.0163 & 1.0125 \\
\hline & 1.0 & 15.118 & 2.3657 & 1.3626 & 1.1503 & 1.0971 \\
\hline & 10.0 & 19.359 & 10.519 & 7.5656 & 7.5863 & 7.6016 \\
\hline \multirow[t]{4}{*}{ \pm 0.5} & 0.01 & 14.363 & 2.0371 & 1.0886 & 1.0266 & 1.0187 \\
\hline & 0.1 & 14.364 & 2.0365 & 1.0915 & 1.0147 & 1.0127 \\
\hline & 1.0 & 15.066 & 2.2155 & 1.3451 & 1.3329 & 1.3326 \\
\hline & 10.0 & 18.505 & 9.9856 & 6.9315 & 6.9807 & 6.9815 \\
\hline \multirow[t]{4}{*}{ \pm 0.8} & 0.01 & 12.776 & 1.8298 & 1.0580 & 1.0228 & 1.0187 \\
\hline & 0.1 & 12.776 & 1.8294 & 1.0608 & 1.0153 & 1.0590 \\
\hline & 1.0 & 12.842 & 2.0520 & 1.3040 & 1.2310 & 1.1956 \\
\hline & 10.0 & 16.554 & 8.8304 & 5.6359 & 6.0836 & 6.0445 \\
\hline
\end{tabular}

\section{B. Initial density distributions}

In order to examine the growth of the instability, we consider two different kinds of initial density distributions. The first, which we term a single-scale perturbation, is of the form

$$
\rho(x, y)=\frac{1}{2}\left[1+A \tanh \left(\frac{1}{L}(y-\epsilon \cos x)\right)\right]
$$

where $A$ is the Atwood number and $L$ is the characteristic thickness of the density layer. The factor $1 / 2$ normalizes the density to lie in the interval from 0 to 1 for the maximum possible Atwood number of 1 . For $L>0$, Eq. (30) describes a continuously stratified interface. As $L$ approaches zero, the layer reduces to a sharp interface with $\epsilon$ as the amplitude of the perturbation. Richtmyer ${ }^{1}$ considered the instability of an interface with an infinitesimal
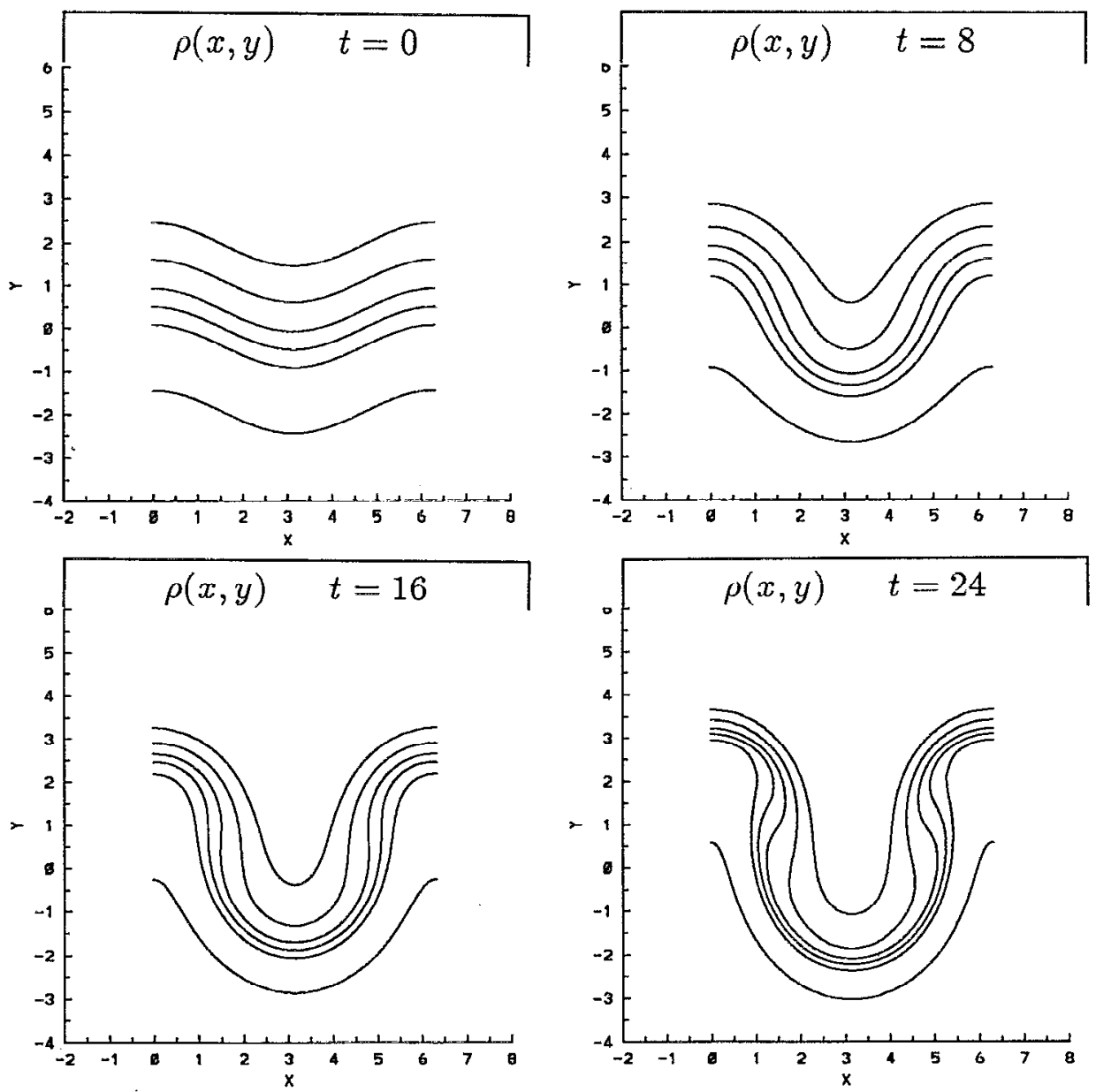

FIG. 1. Time evolution of the density contours for the singlc-seale profile $L=1.0, A=-0.5, \epsilon=0.5, t=0,8,16,24$. The contours are at $\rho=0.26,0.3,0.4,0.5,0.6,0.74$ in that order from top to bottom of each figure. 

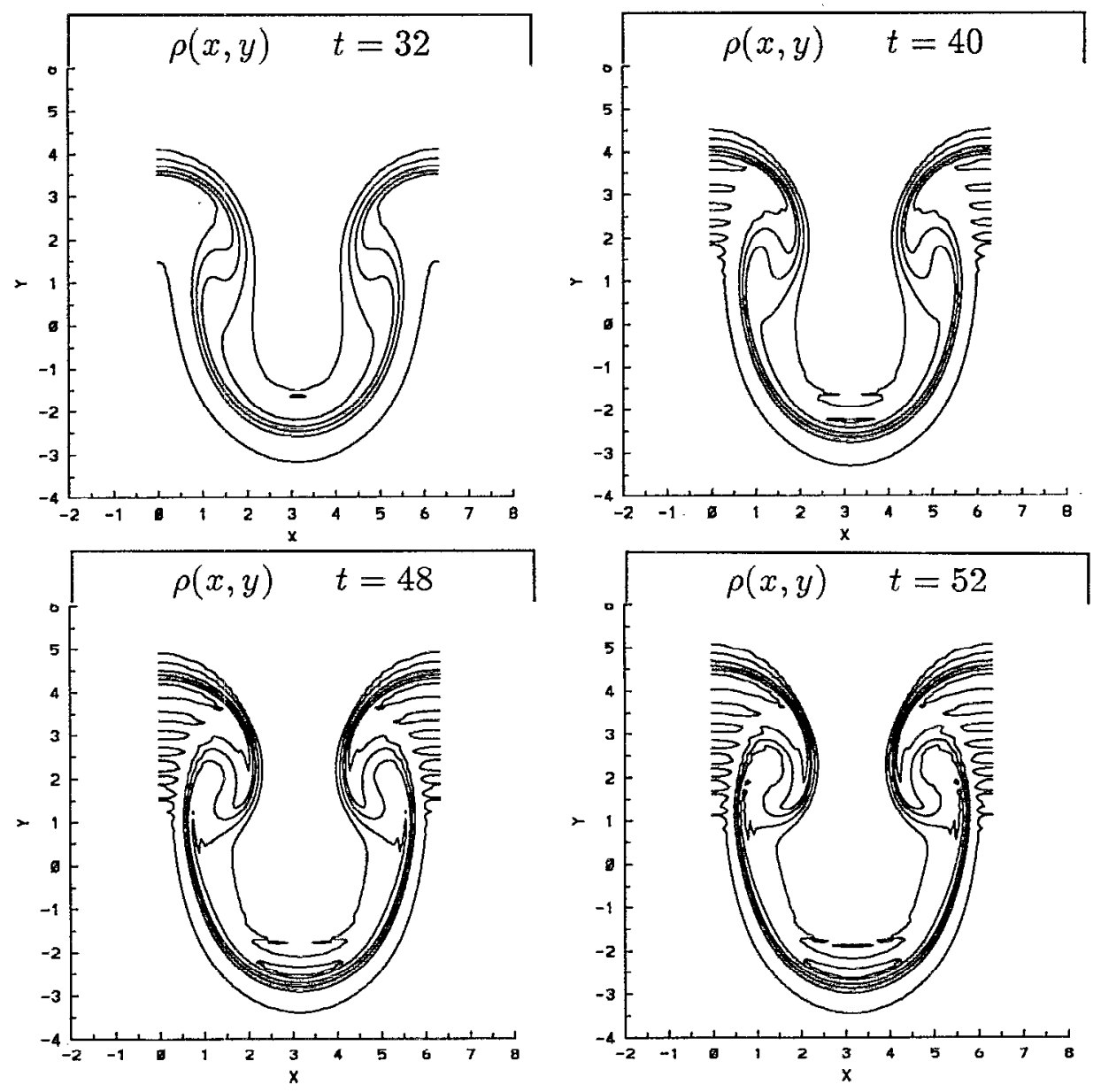

FIG. 2. Time evolution of the density contours for the single-scale profile $L=1.0, A=-0.5, \epsilon=0.5, t=32,40,48,52$. The contours are at $\rho=0.26,0.3,0.4,0.5,0.6,0.74$ in that order from top to bottom of each figure.

perturbation subjected to an impulsive acceleration. With the density profile (30), we can justify our numerical results when $L$ and $\epsilon$ are taken to zero, in that order, for an interface with infinitesimal perturbations. Aside from this consistency chcck, this profile is convenient for the study of nonlinear effects on the growth of the instability of a highly perturbed interface.

In view of the more realistic perturbations that occur in experimental studies, we would like to have some randomness as well as multiple wave numbers present in our initial distribution. To accomplish this we replace $\cos (x)$ in Eq. (30) by the sum,

$$
\sum_{k=1}^{N} e^{\sigma(k 1)^{2}}\left(\frac{1}{2}-r_{k 1}\right) \cos (k x)
$$

or

$$
\sum_{k=1}^{N} e^{-\sigma(k-1)^{2}}\left[\left(\frac{1}{2}-r_{k 1}\right) \cos (k x)+\left(\frac{1}{2}-r_{k 2}\right) \sin (k x)\right],
$$

where $k$ is the wave number, $r_{k 1}, r_{k 2}$ are random numbers with $0 \leqslant r_{k 1}, r_{k 2} \leqslant 1$, and $\sigma$ is a controlling parameter for the spread of the wave-number distribution.
For the single-scale perturbation, we study the effects of varying the Atwood number $A$, the perturbation amplitude $\epsilon$, and the characteristic length $L$, on the development of the instability. For the multiple-scale calculations, we only consider the role of the Atwood number. We have performed multiscale calculations with six different initial conditions represented by Eqs. (31) and (32).

\section{Frozen field approximation}

Because of the weak nature of the instability, we can assume that the initial flow field caused by the passage of the shock, which is modeled by the action of an impulsive motion, is not altered significantly afterward. The energy deposited at the initial instant serves mainly to increase the mixing of the layer through the Lagrangian motion of Muid particles. Thus at early times we can decouple the equations of motion for the velocity and density fields and consider only the Lagrangian evolution of the density by the initial condition. This "linear theory" or "frozen field approximation" can be used to adequately model the initial evolution of the layer at early times.

Consider the density distribution $\rho_{0}(x, \eta)$ at a partic- 

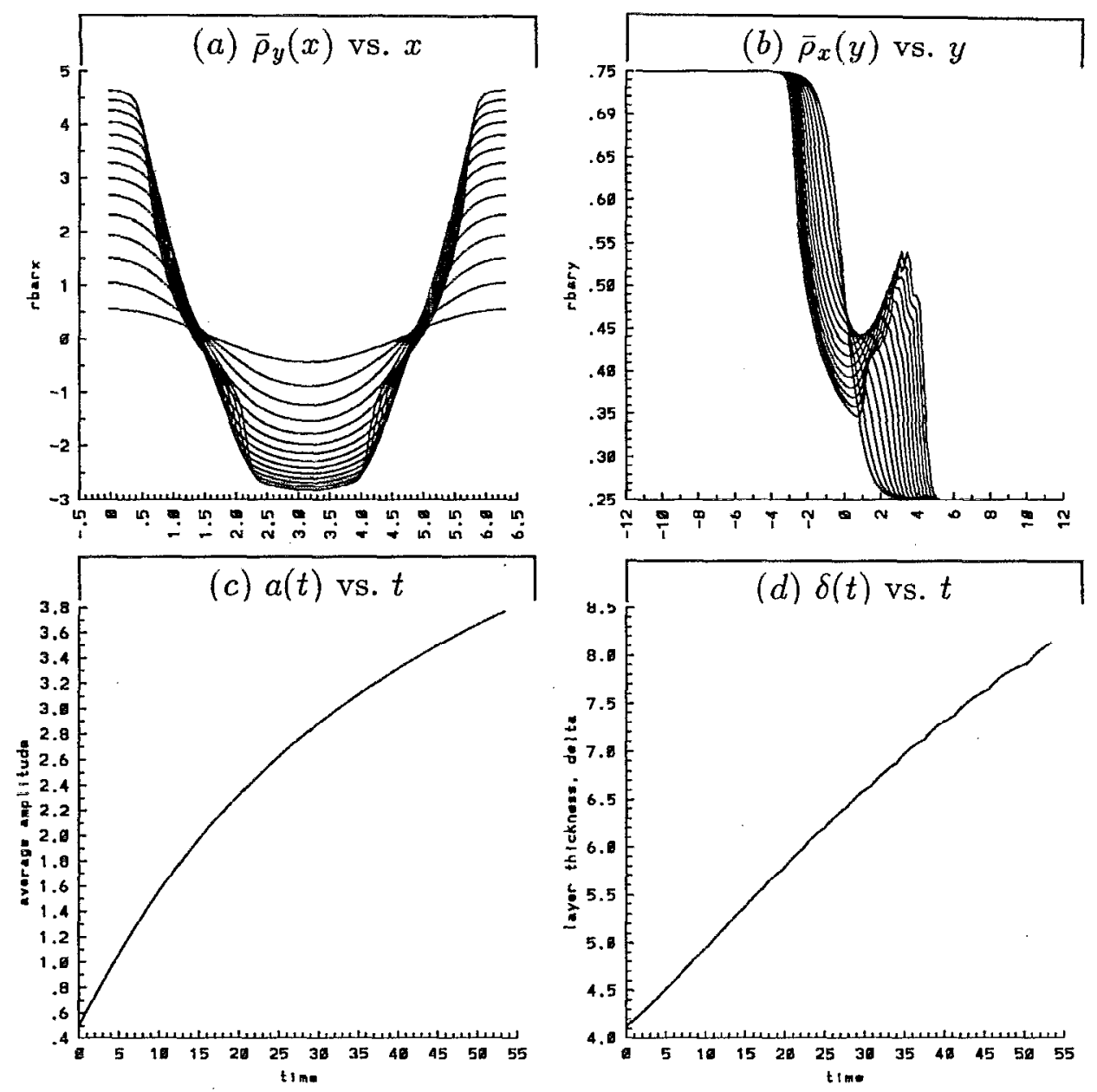

FIG. 3. Time evolution of the average quantities for the single-scale profile $L=1.0, A=-0.5, \epsilon=0.5, t=0-53:(\mathrm{a}) \bar{\rho}_{y}(x)$; (b) $\bar{\rho}_{x}(y)$; (c) average amplitude; and (d) width of the density layer.

ular time $t_{0}$. At a later time $t=t_{0}+\Delta t$, the distribution of the density is $\rho(x, \eta)$. The density at a computational node point $[x(i), \eta(j)]$ at time $t$ is the density of a fluid particle which has arrived at the point $[x(i), \eta(j)]$ from a point having the coordinate $\left(x_{0}, \eta_{0}\right)$, at a time $\Delta t$ earlier. Assume that the velocity of the fluid particle during this motion from time $t_{0}$ to $t$ is constant and equal to the velocity at the node point $x(i), \eta(j)$, (i.e., the redistribution of the density does not affect the flow field significantly). Then the overall algorithm to obtain $\rho(x, \eta)$ from $\rho_{0}(x, \eta)$ is as follows. We compute the velocity at a center $i, j$ of the $p$ cells,

$$
\begin{aligned}
& u_{c}=\frac{1}{2}\left(u_{i j}+u_{i-1, j}\right), \\
& u_{c}=\frac{1}{2}\left(v_{i j}+v_{i, j-1}\right)-V .
\end{aligned}
$$

We then use this velocity to obtain the position $\left(x_{0}, \eta_{0}\right)$ of the fluid particle at time $t_{0}$,

$$
\begin{aligned}
& x_{0}=x_{i j}-u_{c} \Delta t, \\
& \eta_{0}=\eta_{i j}-v_{c} \Delta t .
\end{aligned}
$$

We next search for the grid cell that contains the point $\left(x_{0}, \eta_{0}\right)$. This algorithm is subject to a Courant condition and so we choose the time step so that $\left(x_{0}, \eta_{0}\right)$ will lie very close to the point $[x(i), \eta(j)]$ in one of its four adjacent squares. The final step is to interpolate the density at $\left(x_{0}, \eta_{0}\right)$ using $\rho_{0}$, and assign this value to the point $[x(i), y(j)]$. Let the coordinates of the corners of the square be $\left(x_{l}, \eta_{b}\right),\left(x_{r} \eta_{b}\right),\left(x_{r} \eta_{t}\right)$, and $\left(x_{l}, \eta_{t}\right)$, where the subscripts $l, r, b$, and $t$ denote left, right, bottom, and top respectively. Then $\left(x_{0}, \eta_{0}\right)$ is normalized to

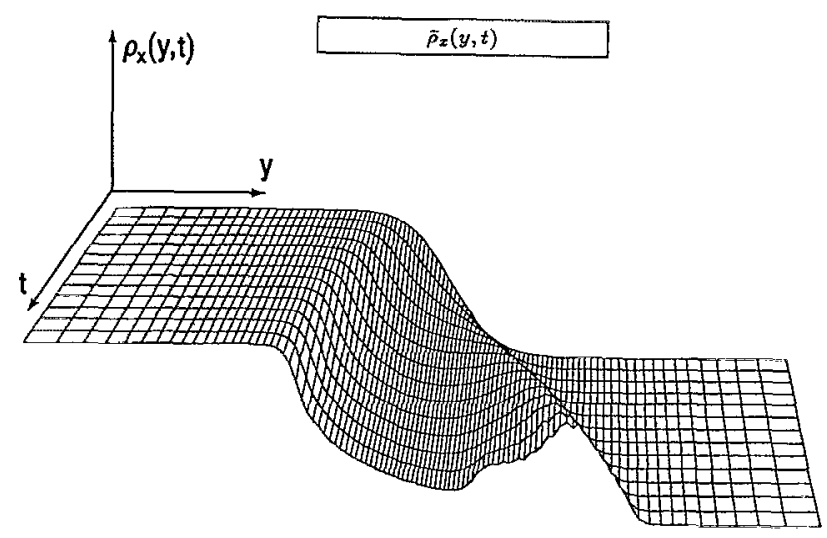

FIG. 4. Three-dimensional surface of $\bar{\rho}_{x}(y, t)$ for the single-scale profile $L=1.0, A=-0.5, \epsilon=0.5$, and $t=0,53$. 

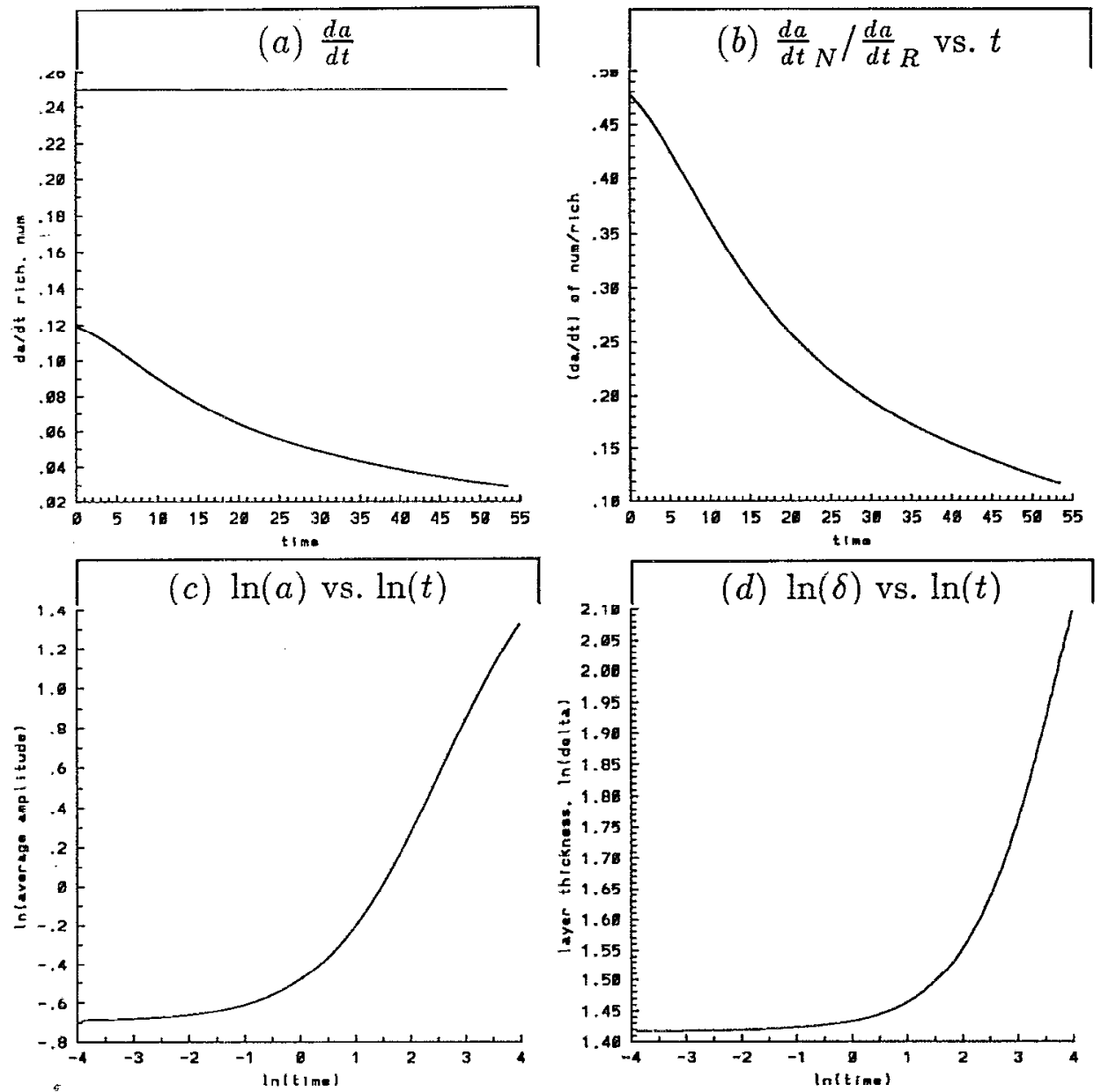

FIG. 5. Time evolution of the average quantities for the single-scale profile $L=1.0, A=-0.5, \epsilon=0.5, t=0-53$ : (a) growth rate $d a / d t$ of the average amplitude, numerical, and Richtmyer theory (straight line); (b) the ratio of the numerical growth rate $d a / d t$ over that predicted by Richtmyer theory; (c) $\ln (a)$ vs $\ln (t) ;(\mathrm{d}) \ln (\delta)$ vs $\ln (t)$.

$$
x_{0 \eta \eta}=\frac{x_{0}-x_{1}}{x_{r}-x_{l}}, \quad \eta_{0 \eta}=\frac{\eta_{0}-\eta_{b}}{\eta_{t}-\eta_{l}} .
$$

The density at $\left(x_{0}, \eta_{0}\right)$ is calculated from the density at the corners of the square using bilinear interpolation,

$$
\begin{aligned}
\rho_{0}\left(x_{0}, \eta_{0}\right)= & \left(1-x_{0 \eta}\right)\left(1-\eta_{0 \eta}\right) \rho_{0}\left(x_{l}, \eta_{b}\right) \\
& +x_{0 \eta}\left(1-\eta_{0 \eta}\right) \rho_{0}\left(x_{r} \eta_{b}\right) \\
& +x_{0 \eta} \eta_{0 \eta} \rho_{0}\left(x_{r} \eta_{t}\right) \\
& +\left(1-x_{0 \eta}\right) \eta_{0 \eta} \rho_{0}\left(x_{l}, \eta_{t}\right) .
\end{aligned}
$$

We will show below that this model provides a good description of the layer dynamics at early times.

\section{Single-scale initial growth rate}

The initial density distribution in Eq. (30) for the single-scale problem has three parameters. The Atwood number $A$ is a measure of the density jump across the stratified layer; similarly $L$ is a measure of the characteristic length of the width of the density layer, and $\epsilon$ is the amplitude of the perturbation. In this subsection, we study the dependence of the initial growth rate of the perturbation as a function of the three aforementioned parameters.

The single-scale cases are solved on a $21 \times 200$ grid, with $\mu=10^{-6}$, and $\Delta t=0.002$. We consider three Atwood numbers $A=-0.2,-0.5$, and -0.8 , five different characteristic lengths $L=0.001,0.01,0.1,1.0$, and 10.0, with four values of the perturbation amplitude $\epsilon=0.01,0.1,1.0$, and 10.0. In Table I, we present the results of this study.

Shown in Table I are the ratios of the initial growth rates of the average amplitude as predicted by the Richtmyer theory over that of numerical simulation. In calculating the equivalent Richtmyer growth rate for a sharp interface,

$$
a=a_{0}, \quad \frac{d a}{d t}=k v_{0} a_{0} A,
$$

we use the initial average amplitude defined in Eq. (25) as $a_{0}$, the velocity $V$ of the flow at infinity as the jump velocity $v_{0}$ of the interface after the impulsive acceleration, and $k=1$. Since the growth rate is an odd function of $A$, the ratios of the growth rates for $\pm A$ are the same. Hence, the 

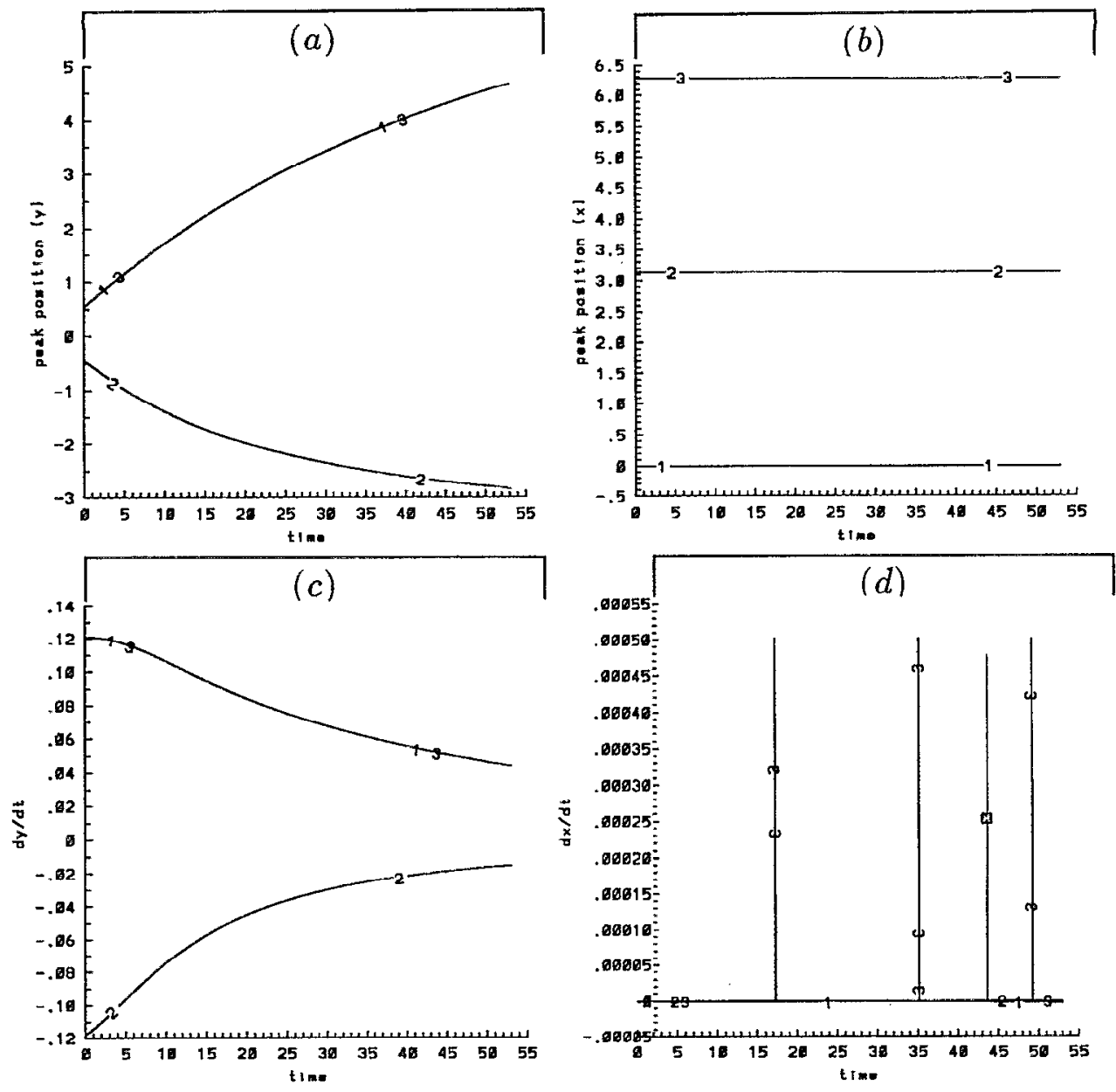

FIG. 6. Time evolution of the positions and velocities of the average peaks in the single-scale profile $L=1.0, A=-0.5, \epsilon=0.5, t=0-53 ;(a) y$; (b) $x$; (c) $d y / d t$; (d) $d x / d t$. The numbers on the curves refer to the peaks on the curves $\bar{p}_{y}(x)$ vs $x$.

results reported here also apply to those for positive Atwood numbers.

For a given $A$, as $L$ approaches zero, i.e., discrete stratification, the ratios approach finite limits which appear to be lower bounds except for the cases of $A=-0.8$, and $\epsilon=10.0$, in which there is a minimum around $L=0.1$. For small perturbation amplitude $\epsilon$, the limit approaches 1.0 from above, which seems to occur for values of $L \leqslant 0.1$. For $0.1<L<0.001$, the ratios of the growth rate do not vary significantly. The ratios increase as $L$ varies from 0.1 to 1.0. For thicker interfaces $(L=1.0$ to 10.0 ), the ratios continue to grow to be of order 10. For $\epsilon=10.0, L=10.0$, and $A=0.2$, the ratio can be as high as 19.4. The corresponding values for $A=0.5$ and 0.8 are, respectively, 18.5 and 16.6. Thus our numerical results are consistent with the predictions of the Richtmyer theory for a discontinuous density gradient. They also confirm the fact that by decreasing the density gradient, one can reduce the growth of the Richtmyer-Meshkov instability as predicted by the linear theory of Saffman and Meiron. ${ }^{2}$ The results also agree qualitatively with the experiments of Brouillette ${ }^{9}$ in which he observes a tenfold reduction in the growth rate for a ratio of thickness to wavelength of around 3. Thus, for a continuous density gradient, the Richtmyer formula
Eq. (39) overpredicts the growth rate.

The behavior of the ratios of the growth rates as a function of the perturbation amplitude $\epsilon$ is similar to that of $L$. The ratios attain a limit from above when $\epsilon$ is less than 1.0. Again the Richtmyer limits are confirmed. For $0.01<\epsilon<1.0$ the ratios do not vary significantly. The ratios of the growth rates increase monotonically with the perturbation amplitude for larger values of $\epsilon$.

\section{E. Late-time behavior for single-scale disturbances}

In this section, we consider the temporal development of the incompressible Richtmyer-Meshkov instability with a continuous density gradient having an initial single-scale perturbation as given in Eq. (30). For $L=1.0$, we have studied cases with combinations from five Atwood numbers $A=-0.05,-0.2,-0.5, \pm 0.8$, and three perturbation amplitudes $\epsilon=0.2,0.5$, and 1.0. For $L=0.5$, we have considered only one case with $A=-0.5$ and $\epsilon=0.2$. Since we are interested in the long-time behavior of the instability, whenever possible we let the simulation proceed up to a time $t$ around 80. It was not always possible to reach this time due to the appearance at late time of small-scale numerical oscillations which indicate loss of resolution. With 

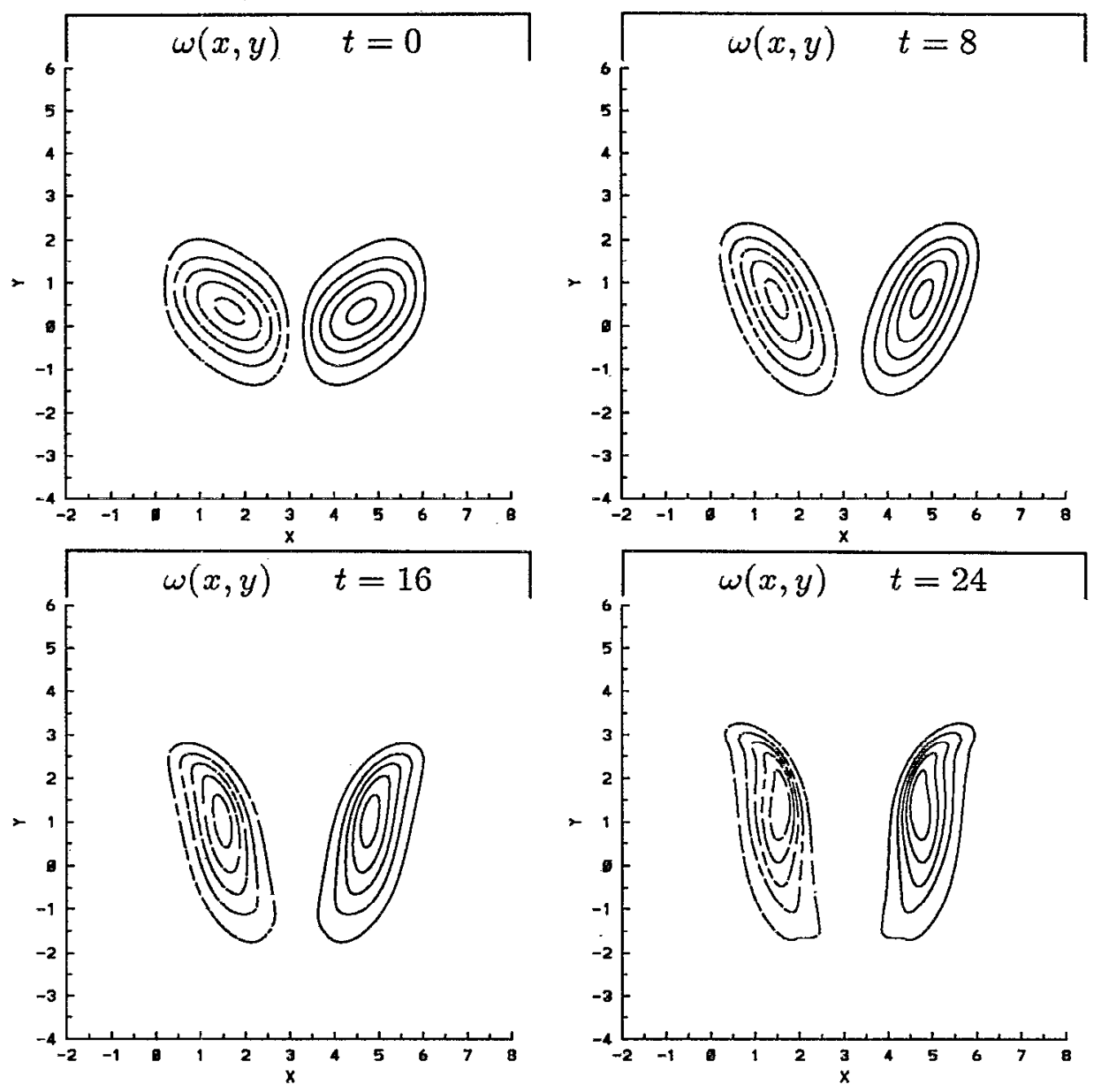

FIG. 7. Time evolution of the vorticity contours for the single-scale profile $L=1.0, A=-0.5, \epsilon=0.5, t=0,8,16,24$. The contours are at $-\cdot-:-0.25$, $-0.20,-0.15,-0.10,-0.05$; and $--: 0.25,0.20,0.15,0.10,0.05$ in that order from the innermost contour line.

an increase in the number of grid points, the calculation can be extended to longer time.

We describe in this section the details of the flow structures that emerge at late times for only one case: $L=1$, $A=-0.5$, and $\epsilon=0.5$. This choice of parameters corresponds to a strong perturbation of the layer and exhibits all of the general features found in our study with other choices for the parameters. A complete account of the cases listed above may be found in the thesis of Pham. ${ }^{13}$

In Figs. 1 and 2 we display the time evolution of the density contours for a simulation with an initial profile given by Eq. (30). Initially the layer behaves much as a discrete interface although with a growth rate that is roughly $1 / 2$ the classical value. However, at $t=24$ we see the emergence of counter-rotating vortices and the formation of a plume at later times. Note that at this point most of the flow is dominated by internal mixing due to the vortices rather than the growth of the amplitude $a(t)$. These features of the flow are reflected in the behavior of the average quantities $\bar{\rho}_{y}(x)$ and $\bar{\rho}_{x}(y)$. In Fig. 3 we display the time evolution of these average quantities as well as the corresponding average amplitude and width of the fluid layer. It is seen that $\bar{\rho}_{y}(x)$ develops additional inflection points. The average amplitude, which is proportional to the peak to trough distance of $\bar{\rho}_{y}(x)$ displays linear behavior initially which is consistent with the growth rates described above but this linear growth does not persist and ultimately slows down, while the width of the fluid layer remains an increasing function of time. Thus, using the average amplitude as a measure of the growth of the instability is somewhat misleading; an examination of $\bar{\rho}_{x}(y)$ reveals that due to the roll-up of the density layer, it loses its monotonicity and develops subsidiary maxima. It will turn out that these maxima are also visible in simulations with multiple-scale perturbations.

The behavior of $\bar{\rho}_{x}(y)$ indicates that on average the entrainment of the heavy fluid into the lighter fluid is faster than the entrainment of lighter fluid into the heavy fluid. This asymmetry in the structures of the spikes of heavy fluid, and the bubbles of light fluid is also observed in the full compressible simulation by Mikaelian ${ }^{18}$ and Youngs, ${ }^{10}$ and has also been observed in the front-tracking calculations of Glimm et al. ${ }^{19}$ Figure 4 displays a perspective view of $\bar{\rho}_{x}(y)$ and reveals this effect clearly.

Detailed information on the average speeds of the spike and bubble is given in Fig. 5 which provides a comparison of the time derivative of the layer amplitude with 

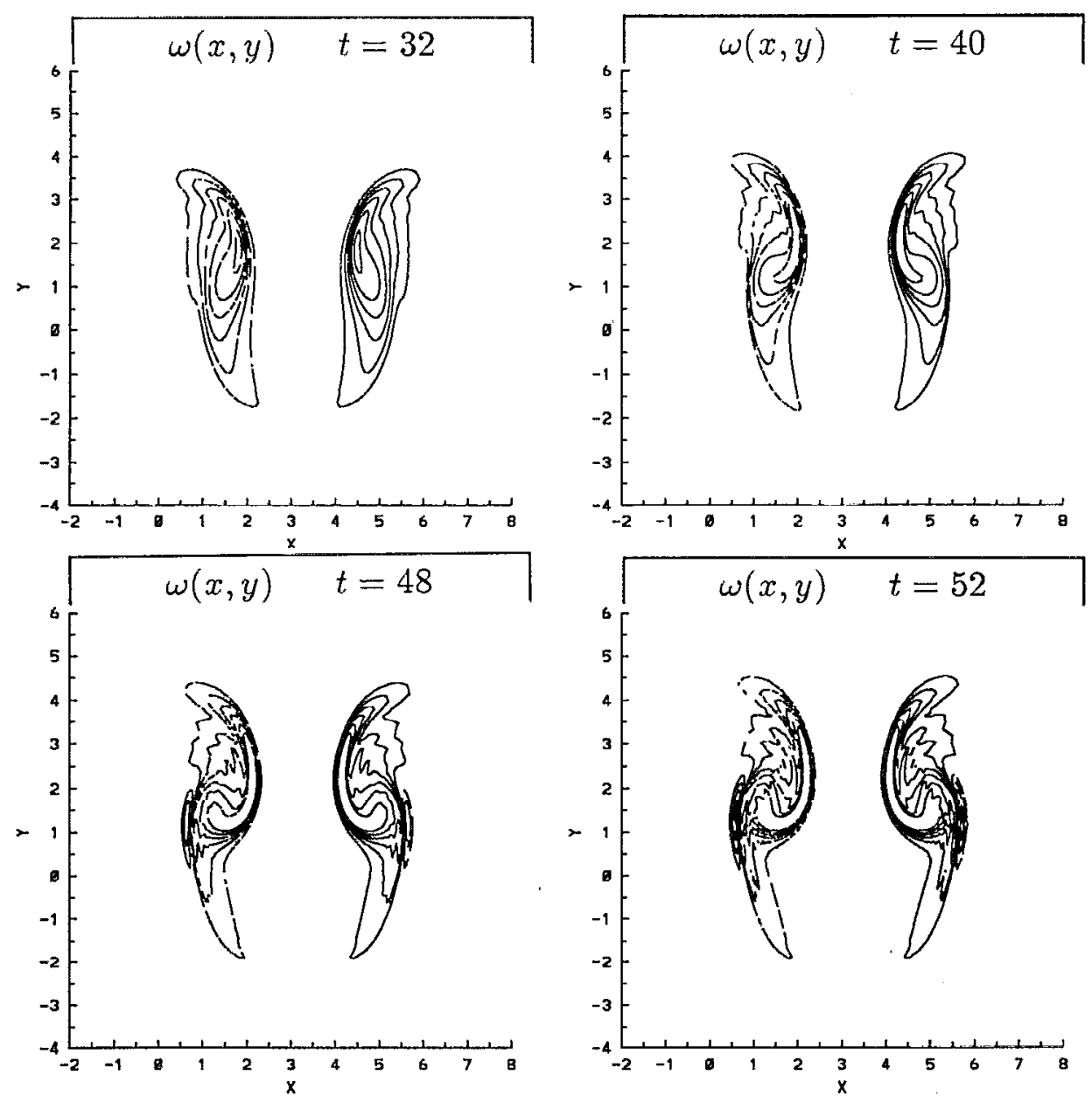

FIG. 8. Time evolution of the vorticity contours for the single-scale profile $L=1.0, A=-0.5, \epsilon=0.5, t=32,40,48,52$. The contours are at $\cdots=$ : $-0.30,-0.25,-0.20,-0.15,-0.10,-0.05$; and $-0.30,0.25,0.20,0.15,0.10,0.05$ in that order from the innermost contour line.

the predictions of the classical Richtmyer theory for this particular Atwood ratio. Note that, in agreement with the results presented earlier, the initial growth rate is overestimated by roughly a factor of 2 relative to the Richtmyer result. As time increases, the instantaneous growth rate is reduced further due to the formation of the roll-up. The moderate to late time behavior of this particular initial condition is clearly not in accord with linear theory since one would expect in this case that $d a / d t$ would remain constant over some period of time. This is due to the strong perturbation inherent in this initial condition. Our results for smaller values of $\epsilon$ are consistent with linear behavior at moderate times.

In general we observe that for small $A$ and $\epsilon$ the speeds of the bubble and spike are equal and opposite as required from linear theory. The light fluid penetrates the heavier fluid at the same rate as the heavier fluid penetrates the light. As seen in Fig. 3, at higher values of $A$ and $\epsilon$ nonlinearity enhances the deceleration of the bubbles of light and heavy fluid. This effect is also clearly seen in Fig. 6 where we plot the positions of the maximum and minimum of $\bar{\rho}_{y}(x)$ corresponding to the tips of the bubble and spike versus time.

The time evolution of the vorticity for this case is displayed in Figs. 7 and 8. The initial vorticity deposited by

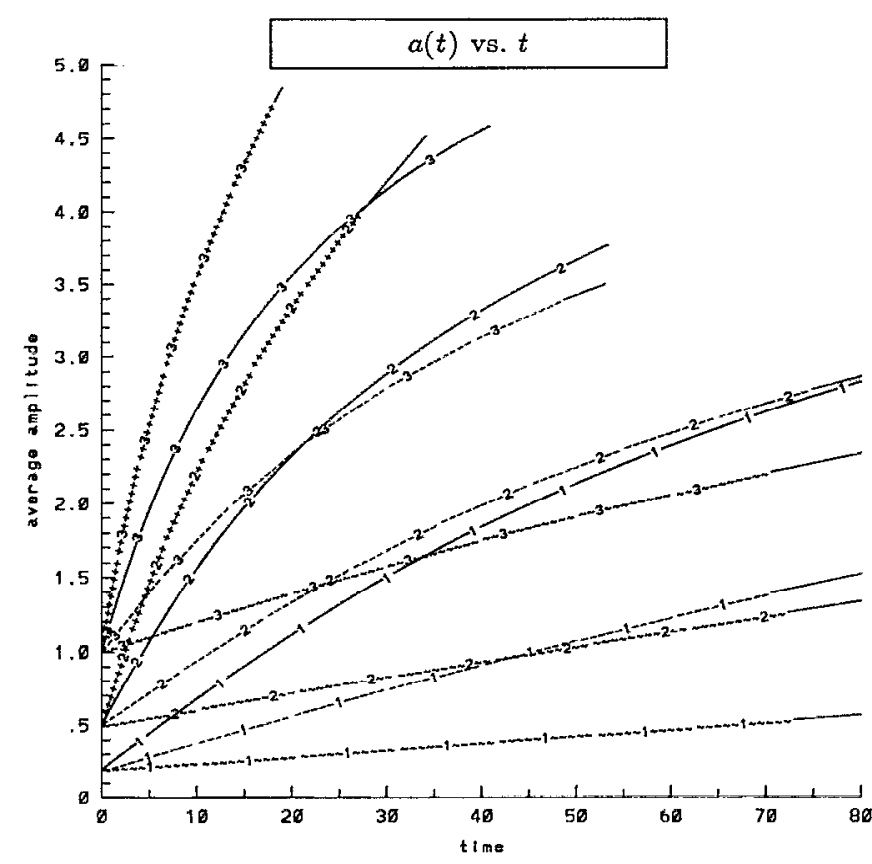

FIG. 9. Combined time evolution of the average amplitude for the singlescale problem $L=1.0: \sim, A=-0.05 ;--, A=-0.2 ;-, A=-0.5$; ,$+++ A=-0.8$. The numbers 1,2 , and 3 on the curves refer to the values of $\epsilon=0.2,0.5$, and 1.0 , respectively. 


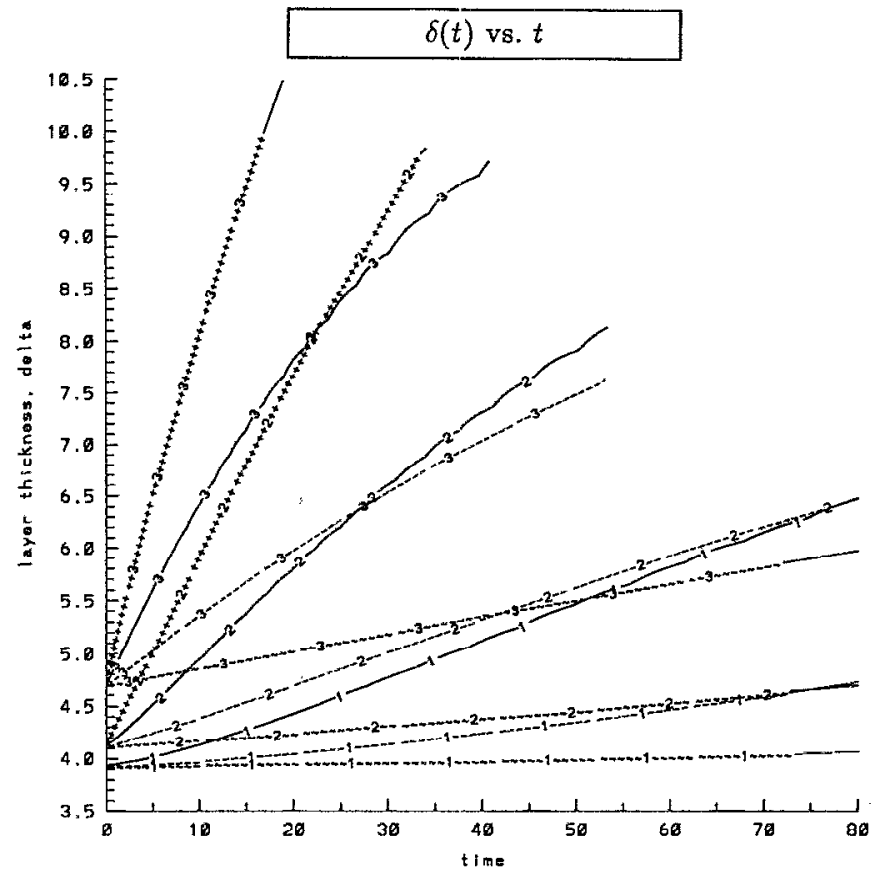

FIG. 10. Time evolution of the width $\delta$ of the stratified layer for the single-scale problem $L=1 ; \sim \sim, A=-0.05 ; \ldots, A=-0.2 ; \square, A$ $=-0.5 ;+++, A=-0.8$. The numbers 1,2 , and 3 on the curves refer to the values of $\epsilon=0.2,0.5$, and 1.0 , respectively.

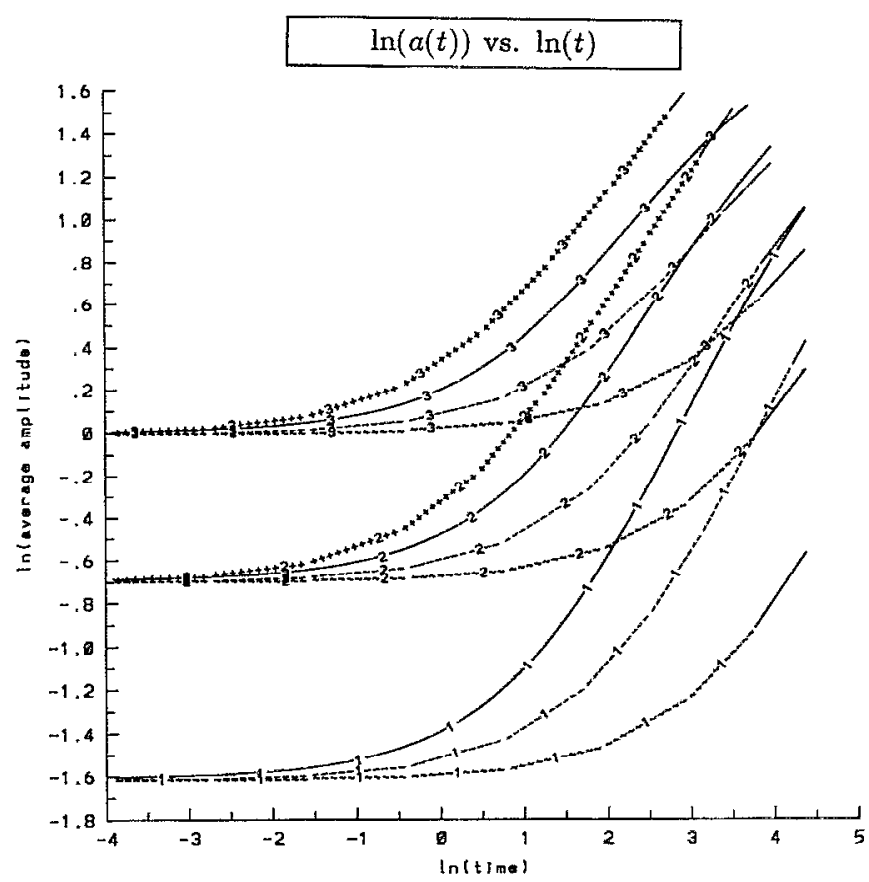

FIG. 11. Combined results of $\ln [a(t)]$ vs $\ln (t)$ for the single-scale problem $L=1: \sim \sim, A=-0.05 ;--, A=-0.2 ;-, A=-0.5 ;+++, A$ $=-0.8$. The numbers 1,2 , and 3 on the curves refer to the values of $\epsilon=0.2,0.5$, and 1.0 , respectively.

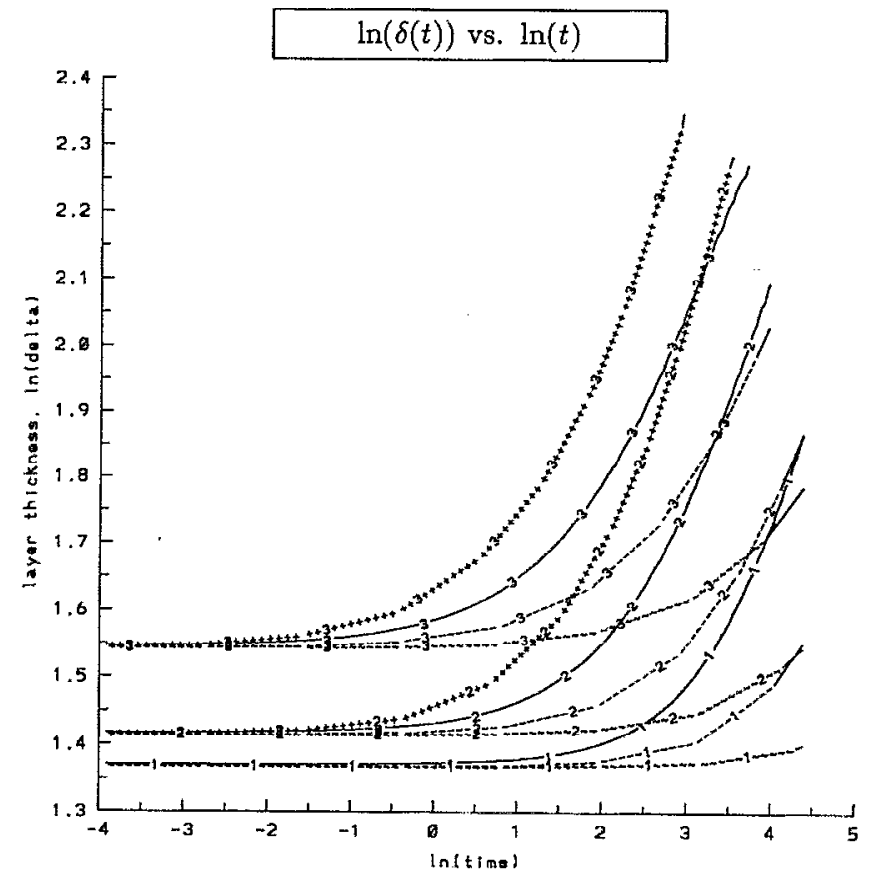

FIG. 12. Combined results of $\ln$ (width of stratified layer $\delta$ ) vs $\ln (t)$ for the single-scale problem $L=1: \sim \sim, A=-0.05 ;--, A=-0.2 ;-, A$ $=-0.5 ;+++, A=-0.8$. The numbers 1,2 , and 3 on the curves refer to the values of $\epsilon=0.2,0.5$, and 1.0 , respectively.

the impulse consists of a pair of counter-rotating vortices which drive the instability. It is this vorticity distribution which is determined through the Saffman-Meiron analysis, and as will be evident below, determines much of the earlytime behavior. As time increases elongation of the vortex structures is observed. This elongation also plays a role in determining the late-time behavior of multiscale perturbations.

The combined results of our single-scale simulations for $L=1$ are shown in Figs. 9 and 10. In Fig. 9 we plot the average amplitude $a(t)$ versus time for Atwood ratios varying from -0.05 to -0.8 and initial amplitudes $\epsilon$ of $0.2,0.5$, and 1.0. Note that in general as the density contrast increases, the rate of growth and interpenetration also increase. In Fig. 10 we plot the width of the layer $\delta(t)$ versus time over the same range of amplitudes and density ratios.

The above results are of use in determining whether there exists any simple asymptotic behavior for large times for either the layer amplitude or width. It is well known that due to the constant forcing inherent in single-scale Rayleigl-Taylor instability the amplitude of a discretely

TABLE II. Asymptotic time exponent $\alpha$ in $\delta \sim t^{\dot{\alpha}}$.

\begin{tabular}{llll}
\hline \hline$A$ & $\epsilon=0.2$ & $\epsilon=0.5$ & $\epsilon-1.0$ \\
\hline-0.05 & & & 0.170 \\
-0.2 & & 0.280 & 0.273 \\
-0.5 & 0.384 & 0.366 & 0.304 \\
-0.8 & & 0.483 & 0.423 \\
\hline
\end{tabular}




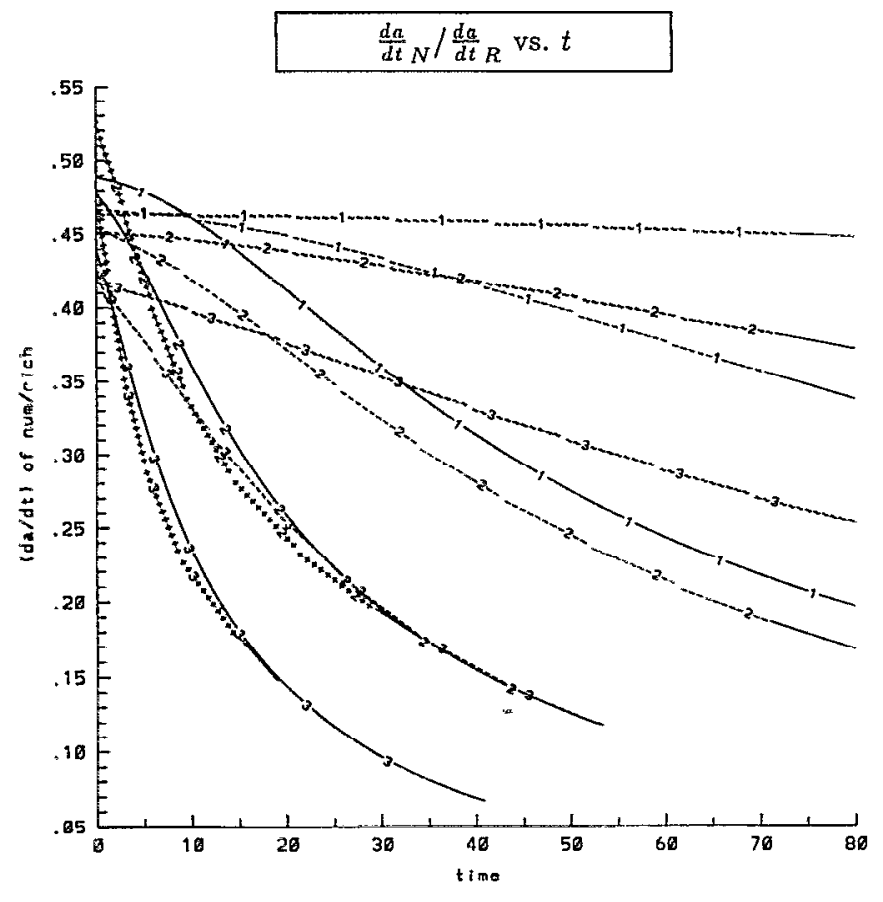

FIG. 13. Time evolution of the ratio of the numerical growth rate over that of Richtmyer theory for the single-scale problem $L=1: \sim \sim, A$ $=-0.05 ;--, A=-0.2 ;-, A=-0.5 ;+++, A=-0.8$. The numbers 1,2 , and 3 on the curves refer to the values of $\epsilon=0.2,0.5$, and 1.0, respectively.

stratified fluid will eventually increase as $A g t^{2}$ where $g$ is the effective acceleration regardless of the initial conditions. In Figs. 11 and 12 we replot the data of Figs. 9 and 10 but on logarithmic scales. The results suggest that while it may be possible to infer late-time power-law behavior the exponents are quite clearly dependent on the initial conditions. We have attempted to fit the width of the layer $\delta$ to a power law of the form

$$
\delta \sim t^{\alpha} .
$$

Estimates of the exponent $\alpha$ are indicated for various values of $\epsilon$ and $A$ in Table II. For a given $\epsilon$, the exponent $\alpha$ increases as the density contrast is increased.

A comparison of the growth of the layer with the classical Richtmyer theory is provided in Fig. 13. Here we display the time evolution of the ratio of the rate of growth of the layer $(d a / d t)$ computed numerically to the classical Richtmyer value. For small density contrasts and small initial amplitude it can be seen that there is indeed a range in which linear theory is valid but the growth rate is approximately a factor of 2 less than that predicted for a discrete stratification. Curves corresponding to such linear initial conditions are constant over some time interval. As the density contrast is increased, however, the flow enters a nonlinear regime more quickly.

Finally, we demonstrate that the "frozen field approximation" described in detail above is useful in predicting the behavior of the layer for moderate time even for perturbations in the nonlinear regime. Since the instability is weak, it can be modeled by fixing the initial flow field after the impulsive acceleration, and letting the flow evolve as discussed in Sec. IV C. Using this model, we obtain the solution for $L=1.0, A=-0.5$, and $\epsilon=0.5$. Shown in Figs. 14 and 15 are comparisons of the average quantities $\bar{\rho}_{y}(x)$, $\bar{\rho}_{x}(y)$ with results obtained using the frozen field model. This indicates that the Saffman-Meiron results can actually be used at moderate times. For example, up to time $t=15$, the differences in the average amplitude $a$, and the layer width $\delta$ relative to the actual calculation are small, $4.43 \%$, and $0.62 \%$, respectively. This agreement corresponds to travel of the layer over a distance comparable to 2.5 to 3 layer widths. At lower Atwood ratio, this agreement extends for even longer times. At later times, the model predicts a growth slower than that observed in the actual calculation. The roll-up is more compact and symmetric for the linear case. This results in a more symmetric form for the average quantities $\bar{\rho}_{x}(y)$. The widening of the light fluid globule is not seen and thus a sharper lower peak of $\bar{\rho}_{y}(x)$ is observed.

\section{F. Instability of the multiple-scale problem}

In this section, we study the instability of a smooth stratification possessing a random superposition of transverse modes as given by Eqs. (31) and (32). We have chosen the characteristic thickness $L=1.0$ with amplitude $\epsilon=0.2$. In the thesis of Pham the instability of six different initial multiscale density profiles is studied in detail. Here we shall present results from the fifth density profile described in that study which is described by Eq. (32), although a compilation of Pham's results is included later in our discussion of the scaling behavior of the interface.

For our numerical simulations we have chosen $N=120$ and $\sigma=0.01$. For this value of $\sigma$, wave numbers larger than 61 cause a variation of less than $10^{-16}$ in the density. For this initial condition we use a $151 \times 100$ grid. This yields at least two points per wavelength, which appears to give reasonable resolution of the subsequent flow.

Shown in Figs. 16-18 is the time evolution of the density contours for the initial condition described above with $A=-0.2$ over the time interval $0<t<75$. Past this time, energy conservation was violated to an unacceptable degree indicating inadequate resolution. The density contours reveal clearly the formation of competing plumes at late times. The plumes appear most distinctly at the boundaries of the density layer.

The formation of these plumes is also reflected in the behavior of the horizontal density average $\rho_{x}(y)$ which is used to calculate the layer width. Shown in Fig. 19 is a perspective view of $\bar{\rho}_{x}(y)$ as a function of time. The layer clearly widens as time evolves, but the plumes manifest themselves as small variations superimposed on a monotonically decreasing function. This is essentially due to effect of the roll-up phenomenon on the shape of the density average for single-scale instability discussed above in Sec. IV E.

The plumes are directly connected to the existence of vortex structures that emerge at late times. Shown in Figs. $20-22$ are contours of vorticity. It is seen that the plumes correspond to dipolar regions of vorticity which propagate 

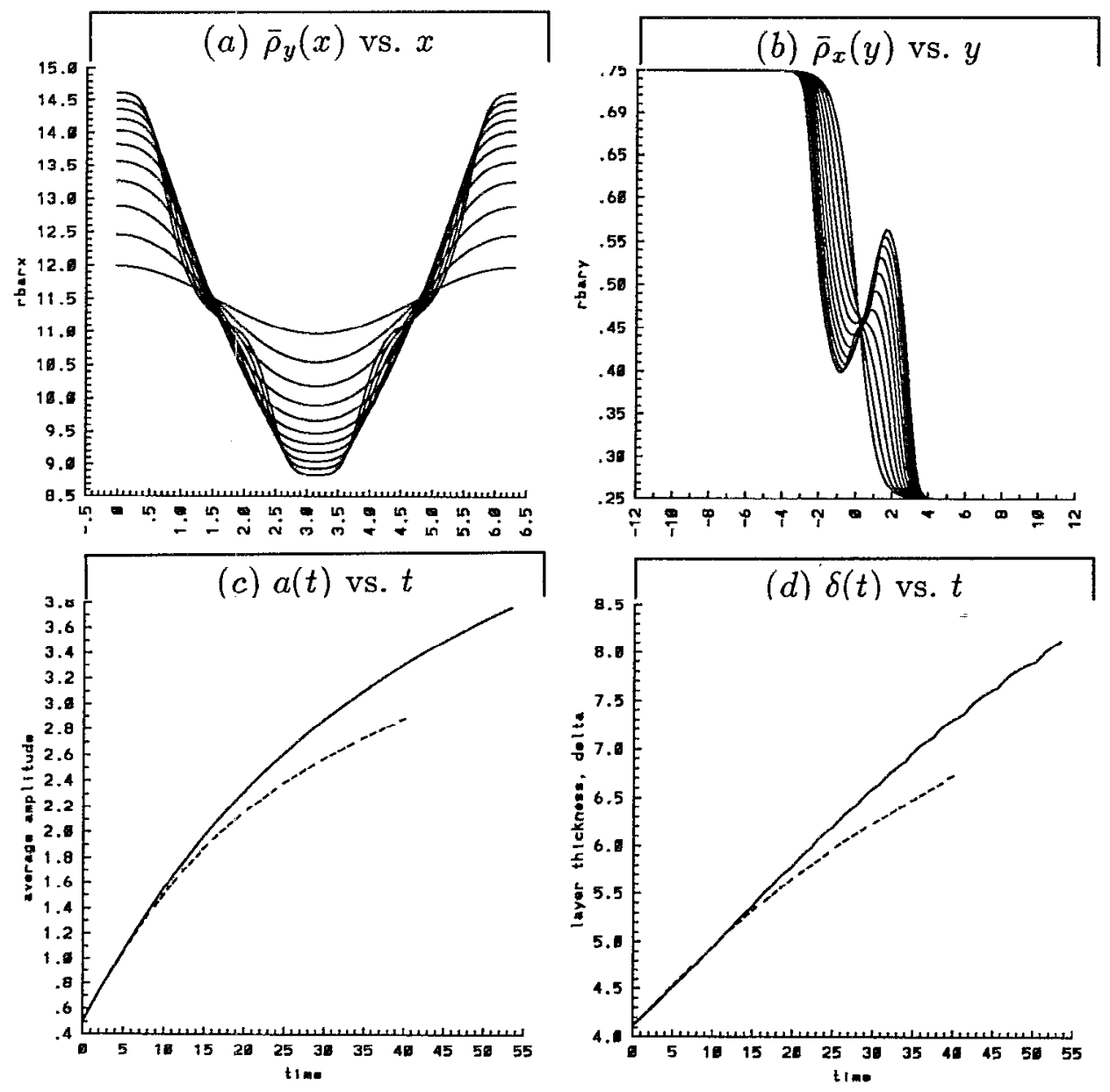

FIG. 14. Time evolution of the average quantities for the single-scale profile $L=1.0, A=-0.5, \epsilon=0.5, t=0-40$ using the linear model: (a) $\bar{\rho}_{y}(x)$; (b) $\bar{\rho}_{x}(y)$. Combined results: _ _ numerical simulation; ---, linear model; (c) average amplitude; and (d) width of the density layer.

through the layer. It is these structures that are ultimately responsible for the widening of the layer and for the irregular appearance of the average density $\bar{\rho}_{x}(y)$.

For higher $A, A=-0.5$ and -0.8 , the characteristics of the flow discussed for $A=-0.2$ are greatly amplified and occur much earlier in time. As in the single-scale perturbation, there exist dipolar regions of vorticity consisting of elongated vortices of equal and opposite strength.

An important issue in this study is the development of a self-similar asymptotic limit independent of the initial random distribution. From the analysis of Barenblatt, ${ }^{12}$ the propagation of a uniform turbulent layer initially deposited in an inviscid homogeneous fluid is self-similar in time. From a dimensional argument, Barenblatt deduced that the layer width scales as

$$
\delta \sim t^{2 / 3}
$$

in the absence of viscosity. Due to the presence of large structures in our problem, there may not be any simple scaling behavior associated with the growth of the mixing layer at late times. Even if such scaling did exist our results indicate that the value of $2 / 3$ for the time exponent is questionable. In an attempt to explore this issue, we plot in Figs. 23 and 24 the time evolution of the average amplitude $a$, and the width $\delta$ of the stratified layer using logarithmic axes. The results are a compilation of six different initial conditions explored by Pham in this thesis. The curves for the amplitude $a$ seem to have a slope of $2 / 3$ while for $\delta$ the slope is close to $1 / 4$. As seen above, the information from the average amplitude $a$ can be misleading in regard to the growth of the layer. In fact it is the quantity $\delta(t)$ which should exhibit growth varying with time as $t^{2 / 3}$, if Barenblatt's theory were valid. Barenblatt's analysis assumes that the layer of turbulence has uniform density. This is clearly not the case here. At best, one might hope that the analysis would be valid at low-density contrasts. However, from the results shown above the scaling exponent for the asymptotic growth of the layer seems to be relatively insensitive to the density contrast at late times.

It is of course possible that the layer has not evolved over a sufficiently long time to display the expected " $2 / 3$ " law. At the end of our calculations the layer has only grown a factor of roughly 2 over its initial size. However, given the emergence of the vortical structures, we find it unlikely that at later times the layer will increase its rate of growth. Barenblatt has also computed the expected scaling exponent in the presence of viscosity and these values are 

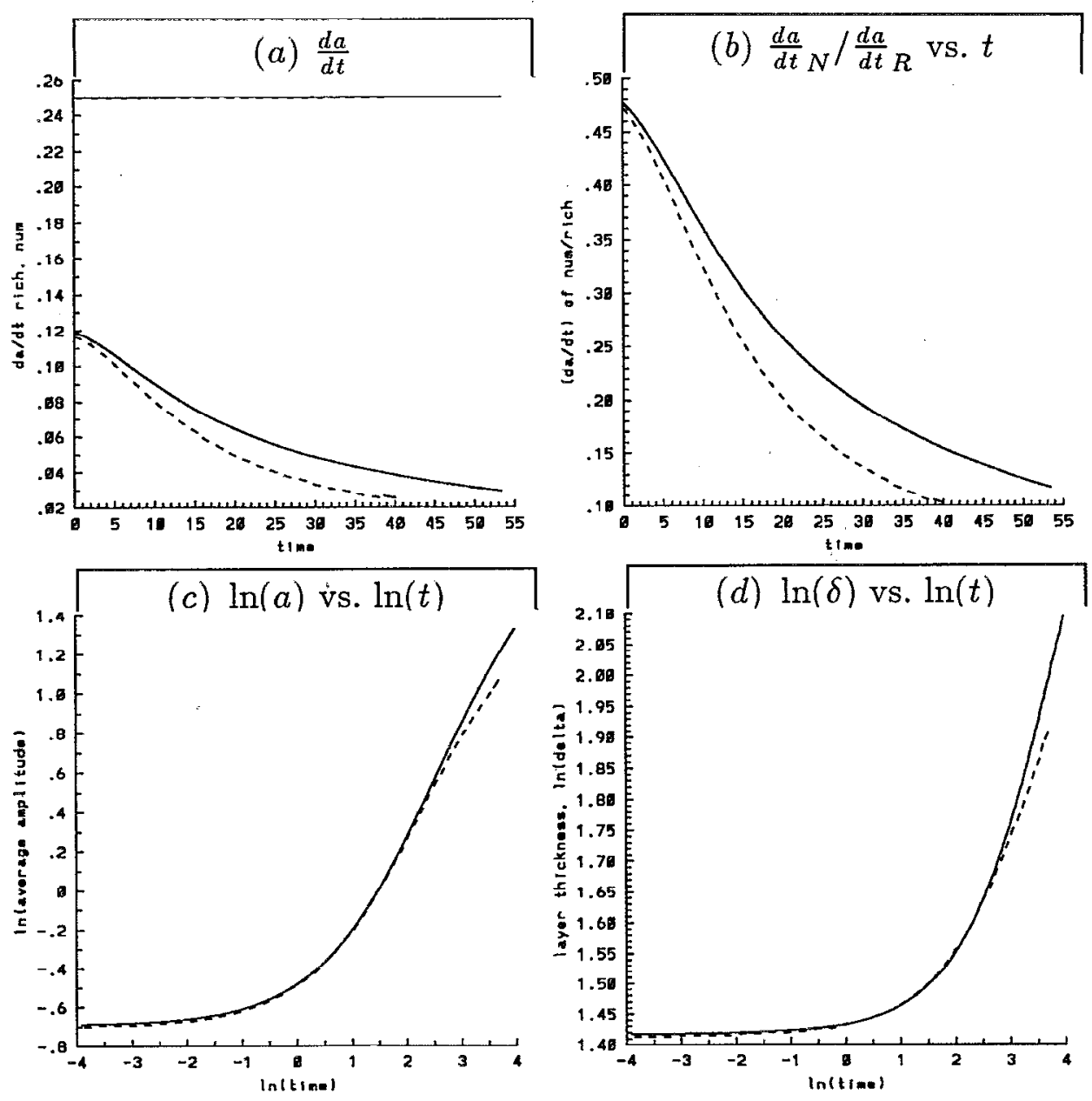

FIG. 15. Time evolution of the average quantities for the single-scale profile $L=1.0, A=-0.5, \epsilon=0.5, t=0-40$. Combined results: $\longrightarrow$, numerical simulation; ---, linear model: (a) growth rate $d a / d t$ of the average amplitude, numerical, and Richtmyer theory (straight line); (b) the ratio of the numerical growth rate $d a / d t$ over that predicted by Richtmyer theory, (c) $\ln a$ vs $\ln t$; (d) $\ln \delta$ vs $\ln t$.

lower than the inviscid value of $2 / 3$. Thus there also exists the possibility that our results reflect some inherent viscosity in the numerical calculation. However, energy is well conserved over the time interval of our calculations and therefore we do not believe that viscosity is a large effect here. Finally, three-dimensional effects which have been ignored here may be responsible for the discrepancy in the exponent. We note, however, that Youngs ${ }^{20}$ has recently presented three-dimensional simulations of RichtmyerMeshkov instability. His results also indicate slower growth of the mixing layer than that given by the Barenblatt theory.

We conclude that while there may be some evidence for scaling behavior in these multiscale simulations, a more elaborate argument which presumably incorporates the effect of structures is necessary to obtain an estimate of the time exponent.

We conclude this section by demonstrating that the frozen field approximation is also of use in determining the early-time behavior of a layer with multiple-scale disturbances. In Fig. 25, we plot the amplitude and layer width versus time for a particular initial condition and compare this with the results of the frozen field approximation. As can be seen from the figure the approximation tracks the growth of the layer quite well at early times. At later times the frozen field model does not account properly for the modification of the density by the vortex structures and underestimates the growth of the layer.

\section{v. CONCLUSIONS}

An extensive simulation of the two-dimensional incompressible Richtmyer-Meshkov instability of a continuously stratified fluid is presented in this study as a model of shock-induced Richtmyer-Meshkov instability. The initial motion after the passage of the shock is assumed to be equivalent to the motion generated by an impulsive acceleration except for the small effect of compressibility. For a small single-scale perturbation on an interface, our numerical growth rates of the instability are the same as those predicted by Richtmyer theory. For a continuous stratification, and similarly for a highly perturbed layer, the Richtmyer formula overpredicts the growth rate. The growth rate varies most strongly with variations of the 

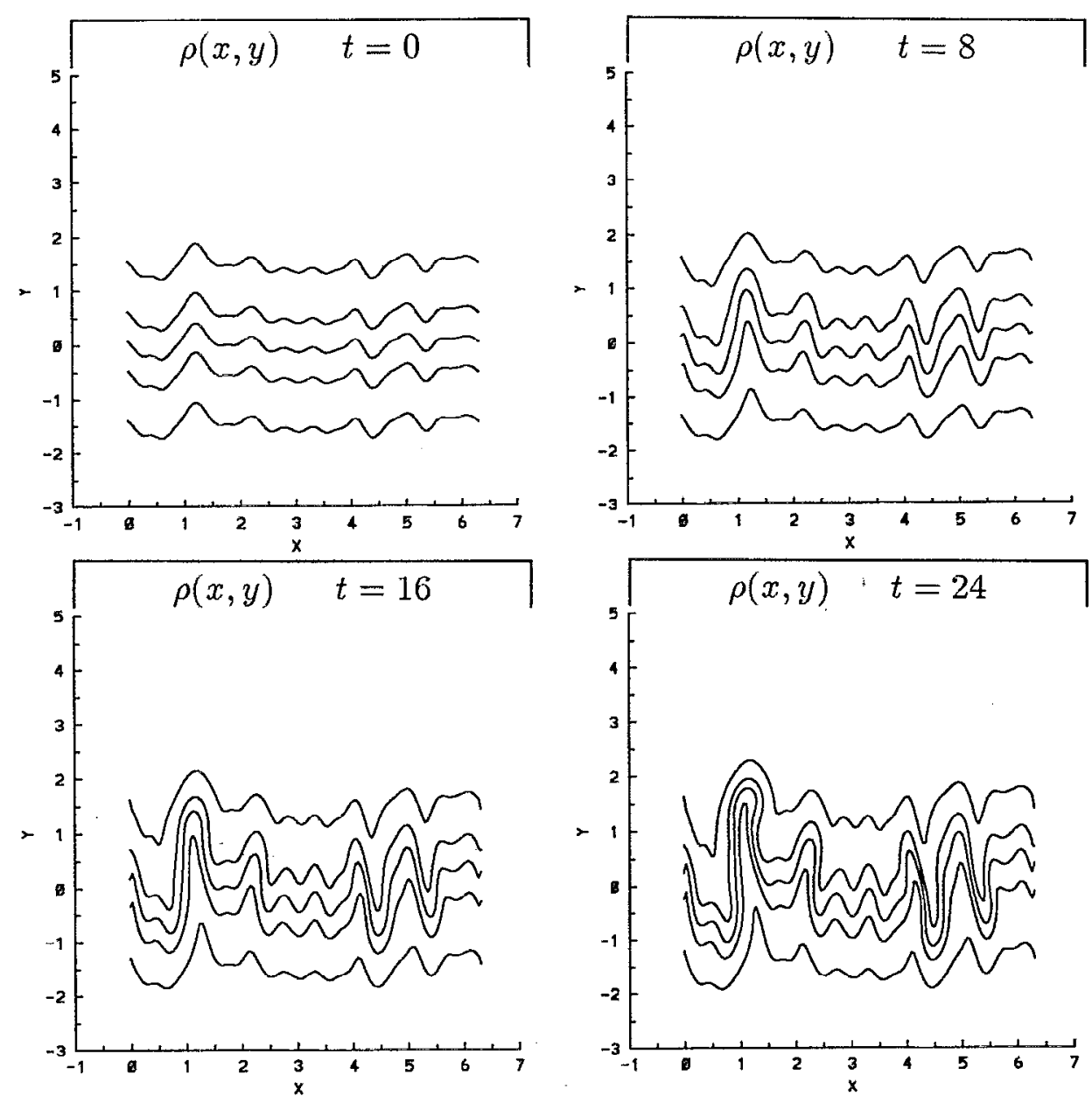

FIG. 16. Time evolution of the density contours for the fifth initial random profile, $A=-0.2, t=0,8,16,24$. The contours are at $\rho=0.41,0.45,0.50$, $0.55,0.59$.

layer thickness. Variation of the growth rate with amplitude and Atwood ratio are less pronounced.

For the single-scale problem, with $L=1.0$, our simulation covers the range of $A$ from -0.8 to -0.05 , and $\epsilon$ from 0.2 to 1.0. The numerical growth rates for these cases are about half of the Richtmyer results for the sharp profiles with an equivalent jump in density. The rate decreases in time in proportion to the nonlinear effects. The higher the perturbation amplitude and the Atwood number, the greater the reduction in the growth rate $d a / d t$. The initial behavior follows the frozen field approximation over a time equivalent to the traversal of several layer thickness. For single-scale perturbations no self-similar long-time asymptotic behavior is observed for the growth of the layer. This is to be contrasted with the case of Rayleigh-Taylor instability in the single-scale regime. For all cases, we observe the emergence of fluid plumes due to roll-up where the nonlinear effects become significant. This phenomenon causes the overall growth of the layer to decrease, and the initial deposited energy is used to increase the internal mixing of the stratified layer.

For the multiple-scale problem, we observe a weak scaling behavior in the growth of the density layer. The layer thickness $\delta$ seems to scale with $t^{1 / 4}$ in contrast to the $t^{2 / 3}$ power law of Barenblatt even at low Atwood ratio. We believe this is a consequence of the inhomogeneity and anisotropy which is related to the presence of large-scale vortices. The late-time behavior of the instability is dominated by the emergence of plumes of fluid, once an internal peak reaches the boundaries of the layer. These fluid globules resemble in some ways the nonlinear development of the single-scale problem. The formations of the plumes may therefore correspond to weakly interacting singlescale modes. In this case it may be possible to base a theory of the growth of the layer on these modes. This would be somewhat in the spirit of the bubble approximation used by Zufiria $^{21}$ in his study of Rayleigh-Taylor instability. It is of course possible that three-dimensional effects which are not included in this calculation may exhibit scaling with a power closer to the value of $2 / 3$ at low Atwood ratios. Alternatively, it is possible that plumelike structures also dominate the late-time behavior in three dimensions. This issue is currently under investigation. 

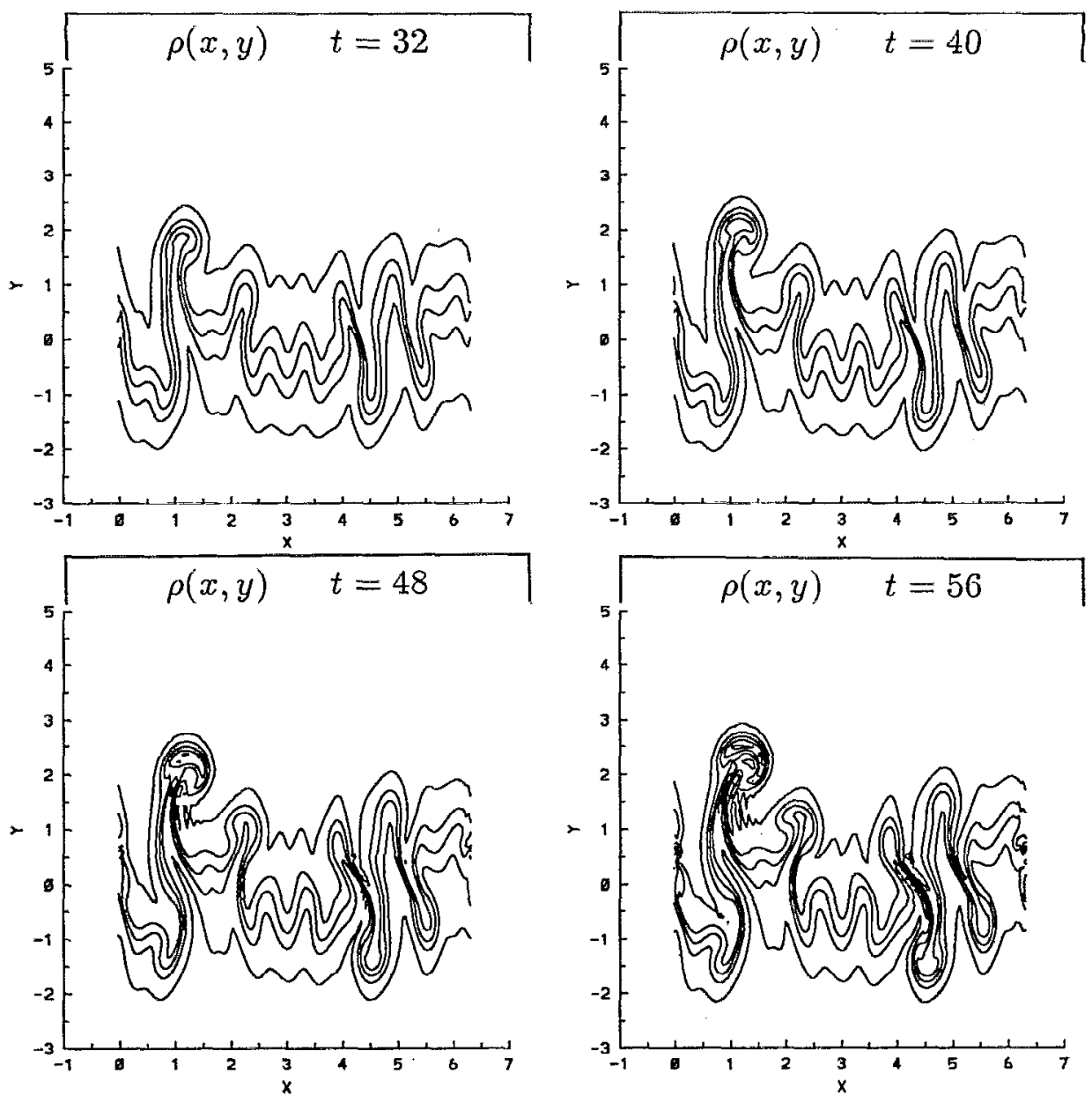

FIG. 17. Time evolution of the density contours for the fifth initial random profile, $A=-0.2, t=32,40,48,56$. The contours are at $\rho=0.41,0.45,0.50$, $0.55,0.59$.

\section{APPENDIX: DETAILS OF THE NUMERICAL SOLUTION}

\section{Stretching functions}

The inversion for $y$ in term of $\eta$ is

$$
y=\frac{1}{\gamma} \ln \sqrt{\frac{1+\eta}{1-\eta}}
$$

The first and second $y$ derivative of a flow variable are transformed as follows:

$$
\begin{aligned}
& \frac{\partial}{\partial y}=\frac{d \eta}{d y} \frac{\partial}{\partial \eta} \\
& \frac{\partial^{2}}{\partial y^{2}}=\left(\frac{d \eta}{d y}\right)^{2} \frac{\partial^{2}}{\partial \eta^{2}}+\frac{d \eta}{d y}\left[\frac{\partial}{\partial \eta}\left(\frac{d \eta}{d y}\right)\right] \frac{\partial}{\partial \eta}
\end{aligned}
$$

where

$$
\begin{aligned}
& \frac{d \eta}{d y}=\gamma\left(1-\eta^{2}\right) \\
& \frac{d \eta}{d y}\left[\frac{\partial}{\partial \eta}\left(\frac{d \eta}{d y}\right)\right]=-2 \gamma^{2} \eta\left(1-\eta^{2}\right) .
\end{aligned}
$$

\section{Discretization of the Poisson equation}

Let $h$ denote the uniform spacing of the grid points in the $x$ direction, and $\Delta \eta$ the spacing in the $\eta$ direction. Equation (17) is discretized at the center of the $p$ cell, and after rearrangement becomes,

$$
\begin{aligned}
& {\left[-\frac{1}{h^{2}}\left(\frac{1}{\rho_{0 i+(1 / 2) j}}+\frac{1}{\rho_{0 i-(1 / 2) j}}\right)\right.} \\
& \left.\quad-n_{u j}^{\prime}\left(\frac{n_{u j}^{\prime}}{\rho_{0 i j+1 / 2}}+\frac{n_{v j-1}^{\prime}}{\rho_{0 i j-1 / 2}}\right)\right] P_{i j} \\
& \quad+\frac{1}{h^{2}} \frac{1}{\rho_{0 i+(1 / 2) j}} P_{i+1 j}+\frac{1}{h^{2}} \frac{1}{\rho_{0 i-(1 / 2) j}} P_{i-1 j} \\
& +\frac{\eta_{u j}^{\prime} \eta_{v j}^{\prime}}{\rho_{0 i j+1 / 2}} P_{i j+1}+\frac{\eta_{u j}^{\prime} \eta_{v j-1}^{\prime}}{\rho_{0 i j-1 / 2}} P_{i j-1}=0,
\end{aligned}
$$

where

$$
\eta_{u}^{\prime}=\left.\frac{d \eta}{d y}\right|_{u} \frac{1}{\Delta \eta},
$$



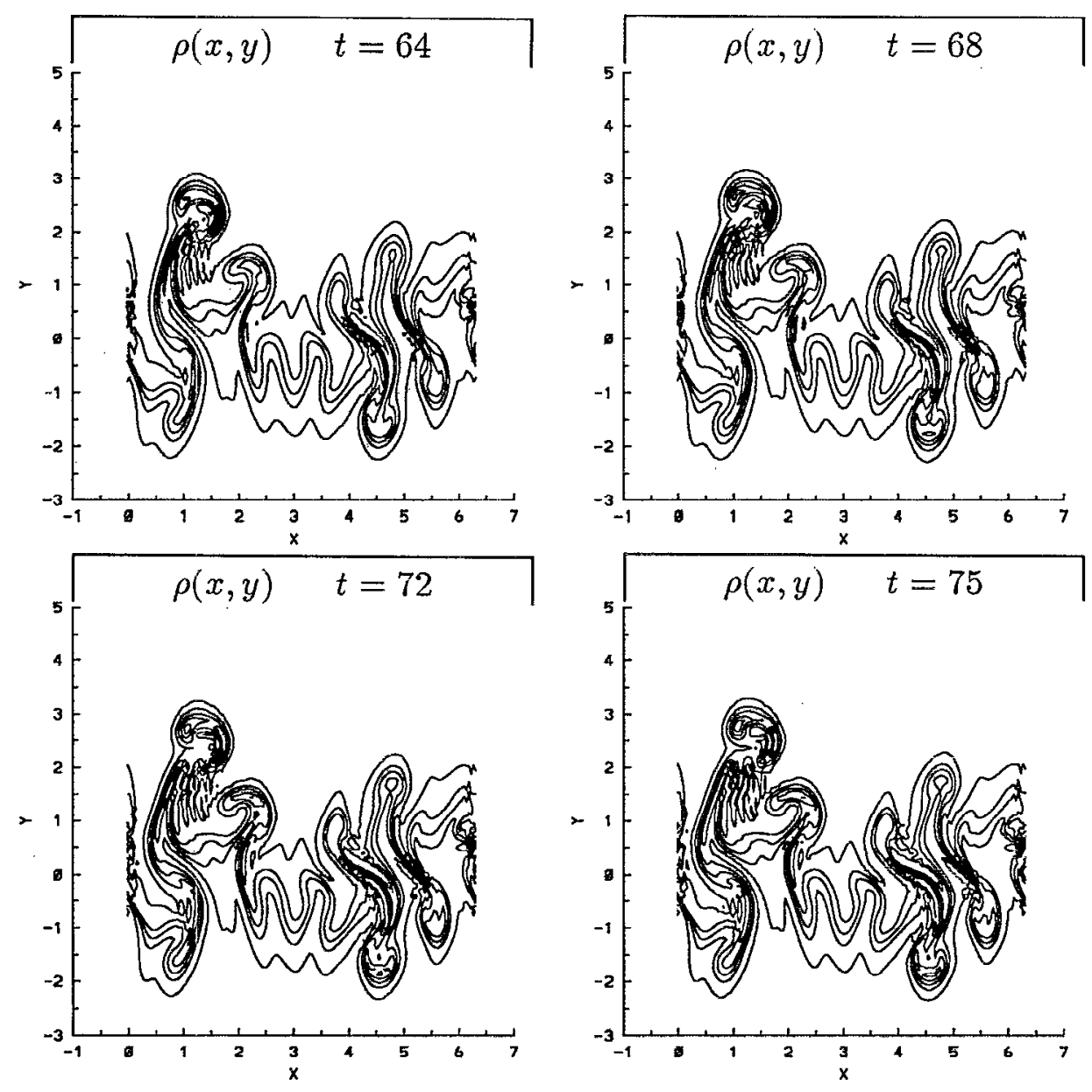
FIG. 18. Time evolution of the density contours for the fifth initial random profile, $A=-0.2, t=64,68,72,75$. The contours are at $\rho=0.41,0.45,0.50$,
$0.55,0.59$.

$$
\eta_{v}^{\prime}=\left.\frac{d \eta}{d y}\right|_{v} \frac{1}{\Delta \eta}
$$

The subscripts $u$ and $v$ denote the evaluation of the derivatives at the center of the $u$ cell and $v$ cell, respectively. Equation (A6) yields a banded matrix, with a band width of twice the number of the grid points in the $x$ direction. This matrix is solved using a band LU decomposition. Since the solution is arbitrary up to a constant, we have the freedom to fix the value of $P$ at one particular grid point. We choose $P_{22}=0$.

\section{Crank-Nicolson time discretization}

The Crank-Nicolson approximation to Eqs. (19)(22) gives

$\frac{\rho^{n+1}-\rho^{n}}{\Delta t}+\frac{1}{2}\left[F\left(\mathbf{u}^{n+1}, \rho^{n+1}\right)+F\left(\mathbf{u}^{n}, \rho^{n}\right)\right]=0$,

$\frac{\mathbf{u}^{n+1}-\mathbf{u}^{n}}{\Delta t}+\frac{1}{2}\left[\mathbf{G}\left(\mathbf{u}^{n+1}, p^{n+1}, \rho^{n+1}\right)+\mathbf{G}\left(\mathbf{u}^{n}, p^{n}, \rho^{n}\right)\right]=0$

$\boldsymbol{\nabla} \cdot \mathbf{u}^{n+1}=0$ where

$$
\begin{aligned}
& F(\mathbf{u}, \rho)=u \frac{\partial \rho}{\partial x}+(v-V) \frac{\partial \rho}{\partial y}, \\
& \mathbf{G}(\mathbf{u}, p, \rho)=\left[G_{1}, G_{2}\right]^{T},
\end{aligned}
$$

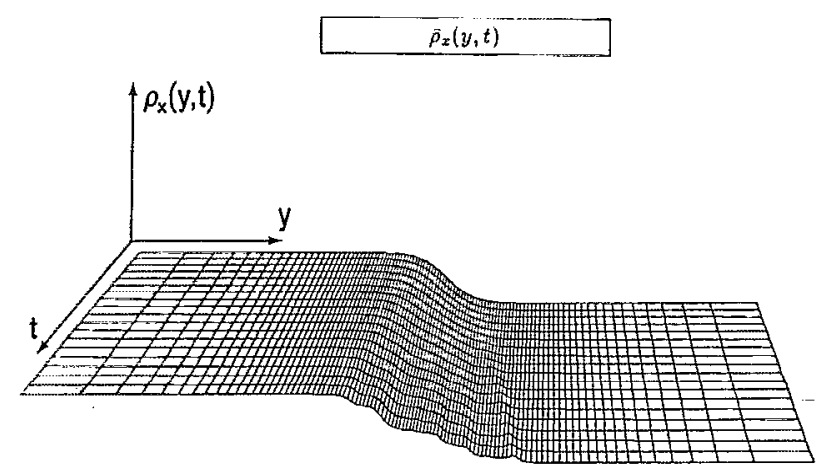

FIG. 19. Three-dimensional surface of $\bar{\rho}_{x}(y, t)$ for the fifth initial random profile, $A=-0.2$ and $t=0,75.4$. 

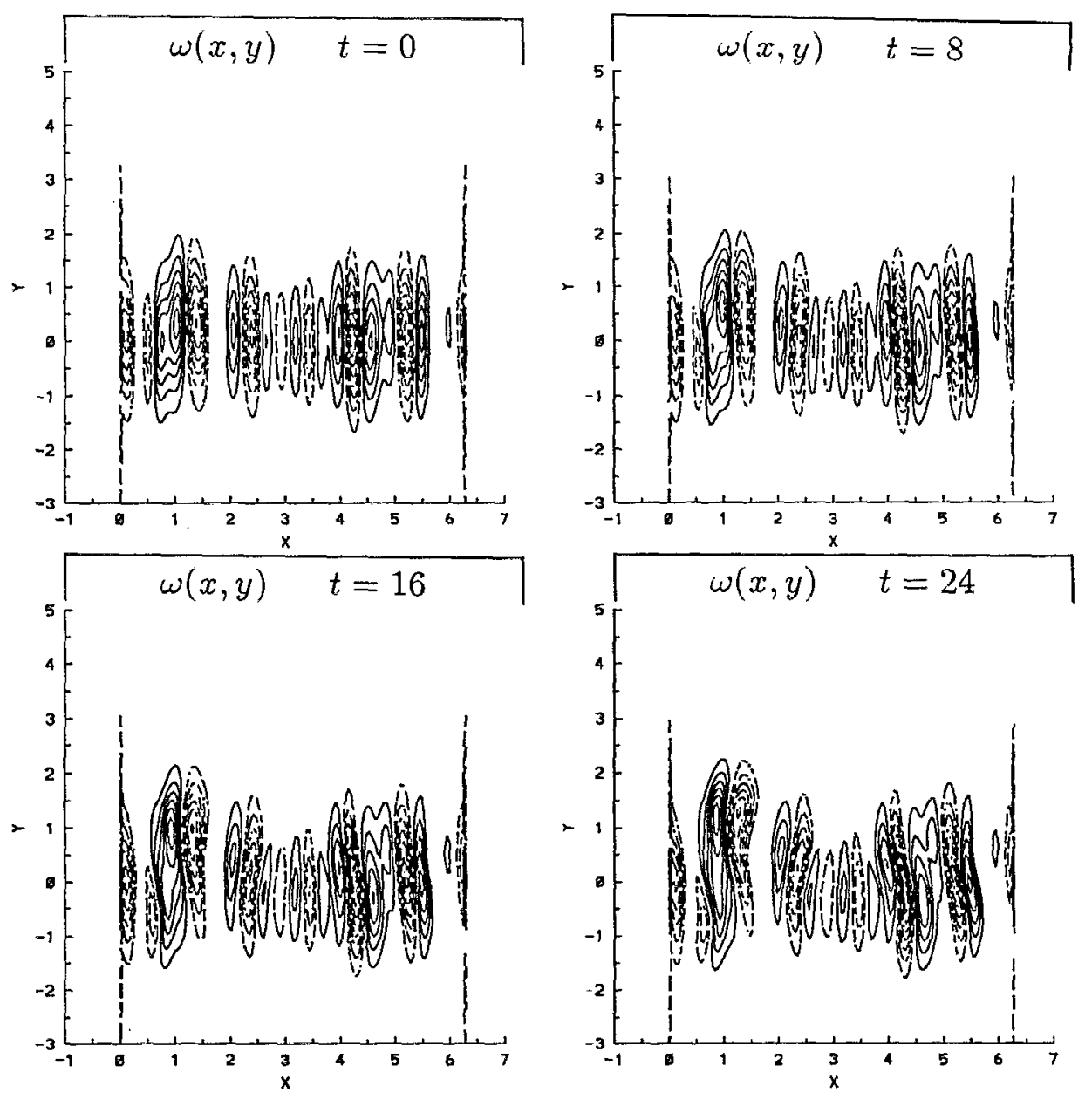

FIG. 20. Time evolution of the vorticity contours for the fifth initial random profile, $A=-0.2, t=0,8,16,24$. The contours are at $-\cdot-:-0.35,-0.30$, $-0.25,-0.20,-0.15,-0.10,-0.05$; and $-0.05,0.10,0.15,0.20,0.25,0.30$ in that order from the innermost contour line.

$$
\begin{aligned}
& G_{1}=\frac{\partial}{\partial x}\left(u^{2}\right)+\frac{\partial}{\partial y}[u(v-V)]+\frac{1}{\rho} \frac{\partial p}{\partial x}-\frac{1}{\rho \operatorname{Re}} \nabla^{2} u, \\
& G_{2}=\frac{\partial}{\partial x}[u(v-V)]+\frac{\partial}{\partial y}\left[(v-V)^{2}\right]+\frac{1}{\rho} \frac{\partial p}{\partial y}-\frac{1}{\rho \operatorname{Re}} \nabla^{2} v .
\end{aligned}
$$

\section{Iterative solution of the equations}

$$
\begin{aligned}
& \text { Let } \\
& \hat{u}^{n+1}=\mathbf{u}^{n+1}-\mathbf{u}^{n}, \\
& \hat{p}^{n+1}=p^{n+1}-p^{n}, \\
& \hat{\rho}^{n+1}=\rho^{n+1}-\rho^{n}, \\
& \alpha=\Delta t / 2,
\end{aligned}
$$

then the above equations become,

$$
\begin{aligned}
& \hat{\rho}^{n+1}+\alpha F\left(\hat{u}^{n+1}+\mathbf{u}^{n}, \hat{\rho}^{n+1}+\rho^{n}\right)=-\alpha F\left(\mathbf{u}^{n}, \rho^{n}\right), \\
& \hat{u}^{n+1}+\alpha \mathbf{G}\left(\hat{u}^{n+1}+\mathbf{u}^{n}, \hat{p}^{n+1}+p^{n}, \hat{\rho}^{n+1}+\rho^{n}\right) \\
& \quad=-\alpha \mathbf{G}\left(\mathbf{u}^{n}, p^{n}, \rho^{n}\right),
\end{aligned}
$$

$$
\nabla \cdot \hat{u}^{n+1}=0 .
$$

This nonlinear set of equations for the unknown variables with the superscript $n+1$ at the next physical time step is solved by considering it to be the steady solution of the following fictitious pseudotime problem:

$$
\begin{aligned}
& \frac{\partial \hat{\rho}}{\partial \tau}+\hat{\rho}+\alpha F(\hat{u}+\mathbf{u}, \hat{\rho}+\rho)=-\alpha F(\mathbf{u}, \rho), \\
& \frac{\partial \hat{u}}{\partial \tau}+\hat{u}+\alpha \mathbf{G}(\hat{u}+\mathbf{u}, \hat{p}+p, \hat{\rho}+\rho)=-\alpha \mathbf{G}(\mathbf{u}, p, \rho), \\
& \beta \frac{\partial \hat{p}}{\partial \tau}+\nabla \cdot \hat{u}=0,
\end{aligned}
$$

where the superscripts $n$ and $n+1$ have been dropped for simplicity.

The initial and boundary conditions for this problem are

$$
\begin{aligned}
& (\hat{u}, \hat{v}, \hat{p}, \hat{\rho})=0 \quad \text { at } \quad \tau=0, \\
& \hat{u}, \hat{v}, \hat{\rho} \rightarrow 0 \quad \text { as } \eta \rightarrow \pm 1, \quad \tau>0,
\end{aligned}
$$



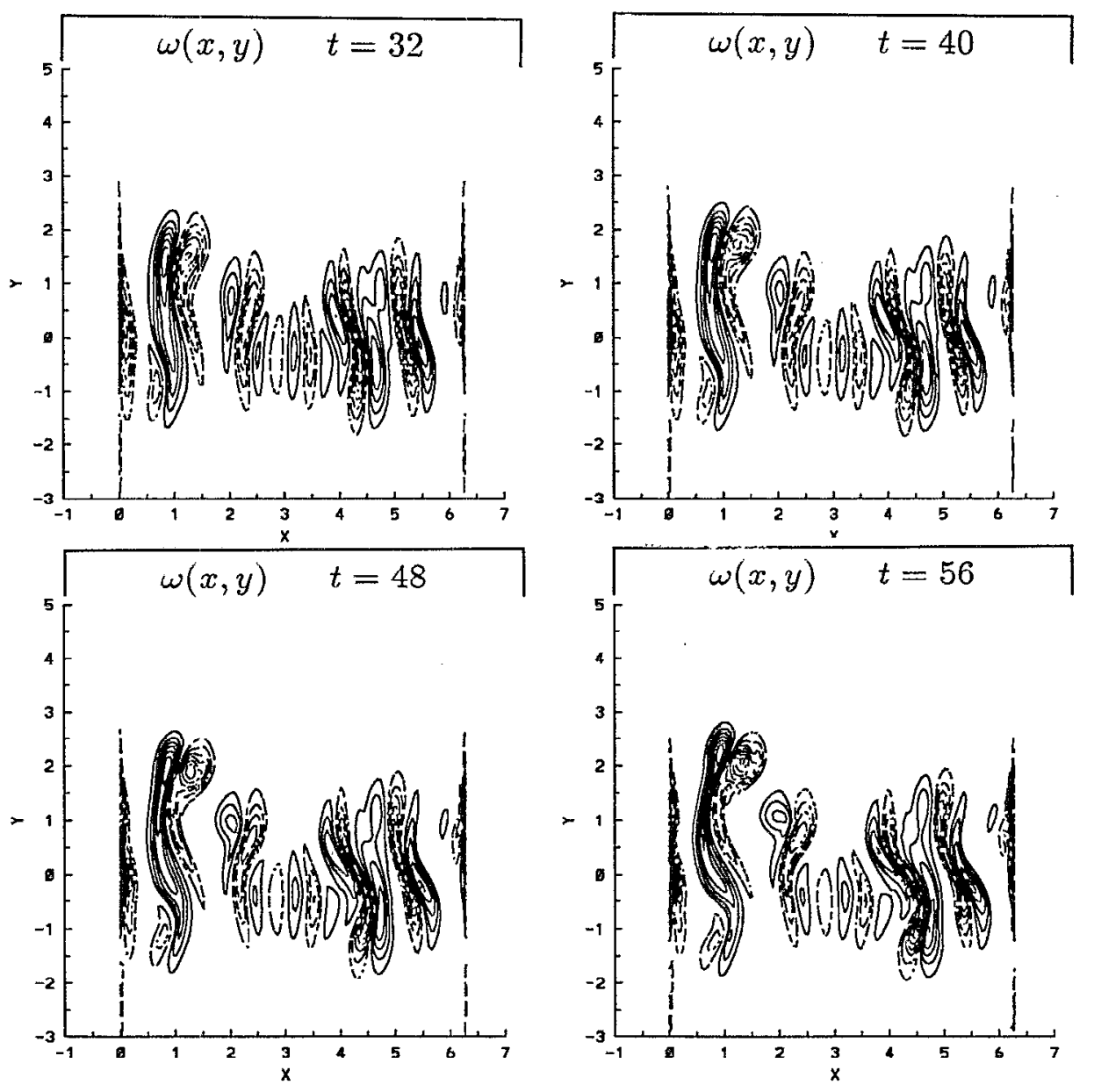

FIG. 21. Time evolution of the vorticity contours for the fifth initial random profile, $A=-0.2, t=32,40,48,56$. The contours are from -0.40 to 0.30 for $t=32,-0.55-0.30$ for $t=40,-0.80-0.30$ for $t=48,56$ with incremental step size of 0.05 . The negative contours are indicated by $-\cdot-$ and positive ones by - The maximum and minimum vorticities are at the centers of the innermost circles.

along with the periodicity of the velocity and the density in the $x$ direction.

Let $k$ denote the $k$ th iteration of the pseudotime problem. Then, one way to solve this fictious system is by the following iterative scheme:

Step 1. For a fixed $i$, along a vertical line of grid points, we discretize the equation for $\hat{v}^{k+1}$ with the $y$ derivatives evaluated at step $k+1$. The $x$ derivatives and all of the nonlinearities are lagged at the pseudotime step $k$. The resulting unknowns can be arranged in the form of a tridiagonal matrix. The pressure in the artificial compressibility equation is coupled with the $v$ momentum equation. Thus, the $v$ momentum equation becomes

$$
\begin{aligned}
\frac{\partial \hat{v}}{\partial \tau}+\hat{v}+\alpha\left(\frac{d \eta}{d y} \frac{\partial}{\partial \eta}[2 \hat{v}(v-V)]+\frac{1}{\hat{\rho}+\rho} \frac{d \eta}{d y} \frac{\partial \hat{p}}{\partial \eta}\right. \\
\left.-\frac{1}{(\hat{\rho}+\rho) \operatorname{Re}}\left\{\left(\frac{d \eta}{d y}\right)^{2} \frac{\partial^{2} \hat{v}}{\partial \eta^{2}}+\frac{d \eta}{d y}\left[\frac{\partial}{\partial \eta}\left(\frac{d \eta}{d y}\right)\right] \frac{\partial \hat{v}}{\partial \eta}\right]\right) \\
=H_{v},
\end{aligned}
$$

where

$$
\begin{aligned}
H_{v}= & -\alpha\left[\frac{\partial}{\partial x}[u(v-V)+(\hat{u}+u)(\hat{v}+v-V)]\right. \\
& +\frac{d \eta}{d y} \frac{\partial}{\partial \eta}\left[2(v-V)^{2}+\hat{v}^{2}\right]+\left(\frac{1}{\rho}+\frac{1}{\hat{\rho}+\rho}\right) \frac{d \eta}{d y} \frac{\partial p}{\partial \eta} \\
& \left.-\frac{1}{(\hat{\rho}+\rho) \operatorname{Re}}\left(\frac{\partial^{2} \hat{v}}{\partial x^{2}}+\nabla^{2} v\right)-\frac{1}{\rho \operatorname{Re}} \nabla^{2} v\right], \quad
\end{aligned}
$$

and

$$
\nabla^{2} \equiv \frac{\partial^{2}}{\partial x^{2}}+\left(\frac{d \eta}{d y}\right)^{2} \frac{\partial^{2}}{\partial \eta^{2}}+\frac{d \eta}{d y}\left[\frac{\partial}{\partial \eta}\left(\frac{d \eta}{d y}\right)\right] \frac{\partial}{\partial \eta} .
$$

We refer to an operation of this type as a "sweep" in order to emphasize the similarity of this method to the wellknown alternating direction implicit (ADI) schemes.

Step 2. Perform a sweep of the density equation in the $y$ direction one-half pseudotime step, $\frac{1}{2} \Delta \tau$ to obtain $\hat{\rho}^{k+1 / 2}$. The density equation for this sweep is,

$$
2 \frac{\partial \hat{\rho}}{\partial \tau}+\hat{\rho}+\alpha(\hat{v}+v-V) \frac{d \eta}{d y} \frac{\partial \hat{\rho}}{\partial \eta}=H_{r 1},
$$



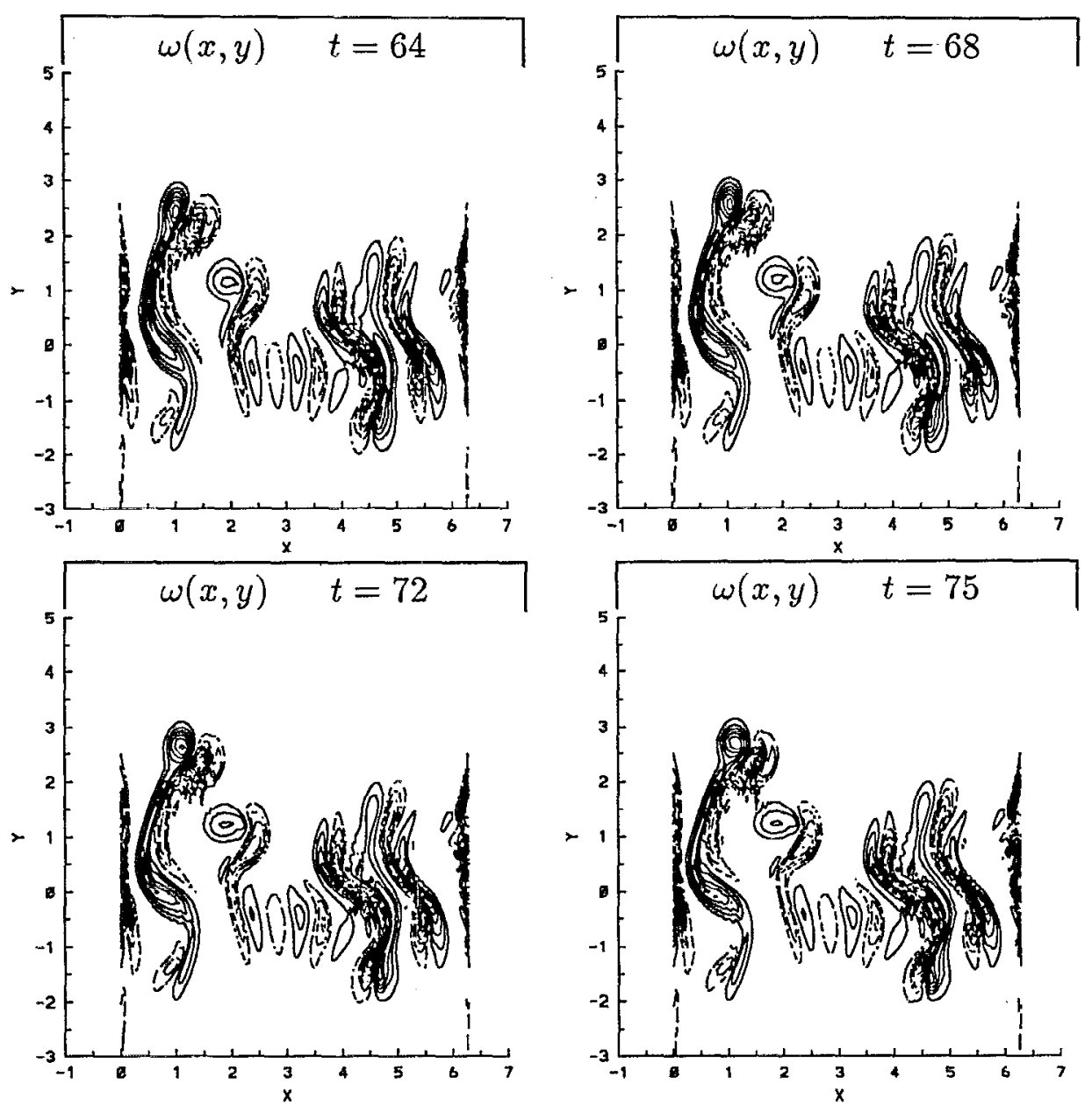

FIG. 22. Time evolution of the vorticity contours for the fifth initial random profile, $A=-0.2, t=64,68,72,75$. The contours are from $-0.60-0.30$ $t=64,-0.65-0.40 t=68,-0.60-0.60 t=72$, and $-0.60-0.70 t-75$ with incremental step size of 0.05 . The negative contours are indicated by $-\cdot-$ and positive ones by

where

$$
\begin{aligned}
H_{r 1}= & -\alpha\left((2 u+\hat{u}) \frac{\partial \rho}{\partial x}+[2(v-V)+\hat{v}] \frac{d \eta}{d y} \frac{\partial \rho}{\partial \eta}\right. \\
& \left.+(\hat{u}+u) \frac{\partial \hat{\rho}}{\partial x}\right) .
\end{aligned}
$$

Step 3. Perform a sweep of the equation for $\hat{u}$ in the $x$ direction. The pressure in the artificial compressibility equation is coupled with the $u$-momentum equation. Only $x$ derivatives are considered and all of the $y$ derivatives and the nonlinearities are lagged. The $u$-momentum equation for this sweep is then

$\frac{\partial \hat{u}}{\partial \tau}+\hat{u}+\alpha\left(\frac{\partial}{\partial x}(2 u \hat{u})+\frac{1}{\hat{\rho}+\rho} \frac{\partial \hat{p}}{\partial x}-\frac{1}{(\hat{\rho}+\rho) \operatorname{Re}} \frac{\partial^{2} \hat{u}}{\partial x^{2}}\right)=H_{u}$

where

$$
\begin{aligned}
H_{u}= & -\alpha\left\{\frac{\partial}{\partial x}\left(2 u^{2}+\hat{u}^{2}\right)+\frac{d \eta}{d y} \frac{\partial}{\partial \eta}[u(v-V)\right. \\
& +(u+\hat{u})(v+\hat{v}-V)]+\left(\frac{1}{\rho}+\frac{1}{\hat{\rho}+\rho}\right) \frac{\partial p}{\partial x} \\
& -\frac{1}{(\hat{\rho}+\rho) \operatorname{Re}}\left[\left(\frac{d \eta}{d y}\right)^{2} \frac{\partial^{2} \hat{u}}{\partial \eta^{2}}+\frac{d \eta}{d y} \frac{\partial}{\partial \eta}\left(\frac{d \eta}{d y}\right) \frac{\partial \hat{u}}{\partial \eta}+\nabla^{2} u\right] \\
& \left.-\frac{1}{\rho \operatorname{Re}} \nabla^{2} u\right\} .
\end{aligned}
$$

Step 4. Perform a sweep in the $x$ direction for the remaining half pseudotime step for the density equation. The density equation is

$$
2 \frac{\partial \hat{\rho}}{\partial \tau}+\hat{\rho}+\alpha(\hat{u}+u) \frac{\partial \hat{\rho}}{\partial x}=H_{r 2},
$$

where 


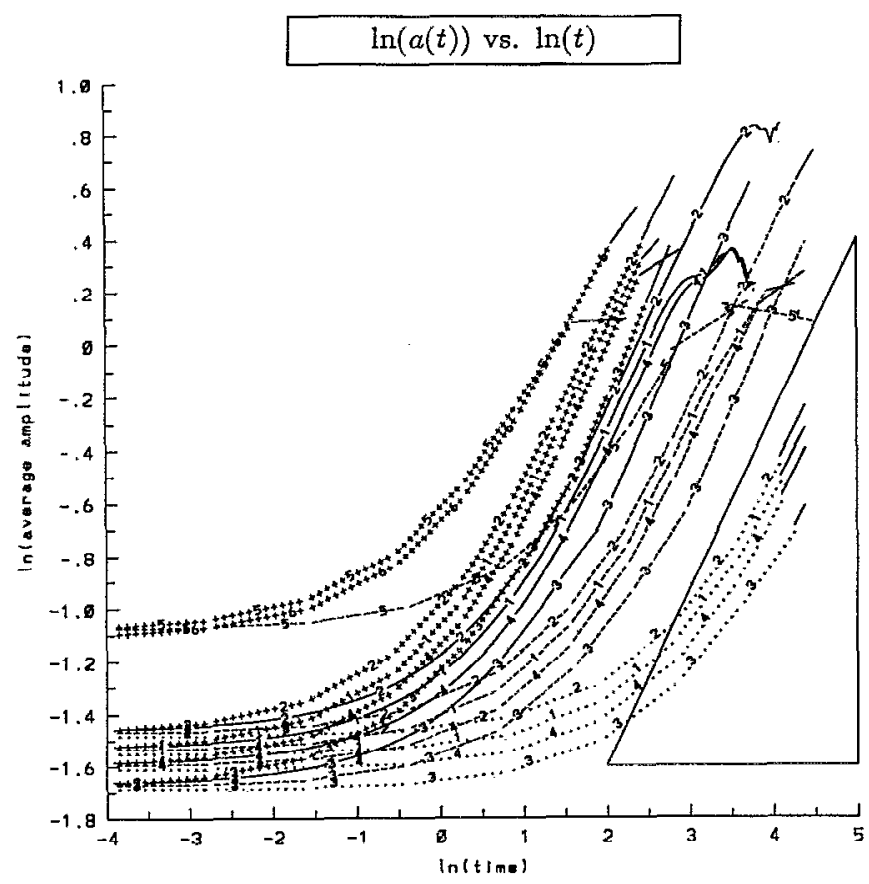

FIG. 23. Combined results of $\ln$ (average amplitude) vs $\ln (t)$ for six different initial random profiles: $\cdots, A=-0.05 ; \ldots, A=-0.2 ;-$, $A=-0.5 ;+++, A=-0.8$. The numbers $1,2,3,4,5$, and 6 on the curves refer to the first, second, third, fourth, fifth, and sixth initial random profiles, respectively. The triangle has slope $2 / 3$.

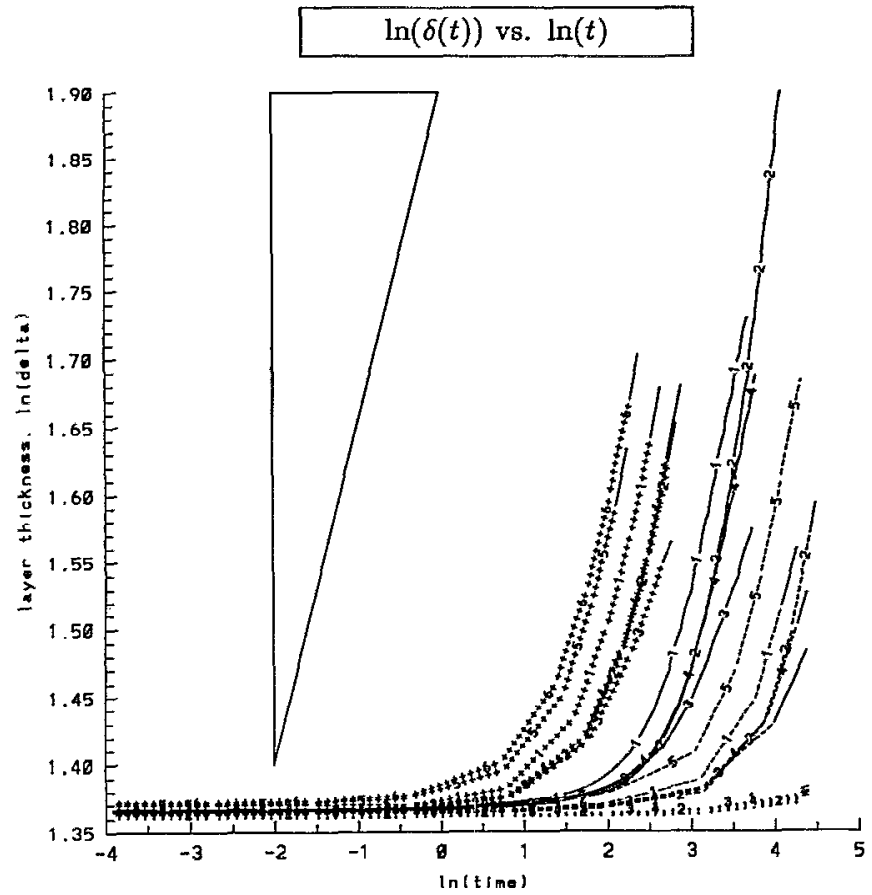

FIG. 24. Combined results of $\ln \delta$ vs $\ln t$ for six different initial random profiles: $\cdots, A=-0.05 ;-\cdots, A=-0.2 ;-, A=-0.5 ;+++, A$ $=-0.8$. The numbers $1,2,3,4,5$, and 6 on the curves refer to the first, second, third, fourth, fifth, and sixth initial random profiles, respectively. The triangle has slope $1 / 4$.

$$
\begin{aligned}
H_{r 2}= & -\alpha\left((2 u+\hat{u}) \frac{\partial \rho}{\partial x}+[2(v-V)+\hat{v}] \frac{d \eta}{d y} \frac{\partial \rho}{\partial \eta}\right. \\
& \left.+(\hat{v}+v-V) \frac{d \eta}{d y} \frac{\partial \hat{\rho}}{\partial \eta}\right) .
\end{aligned}
$$

Step 5. Obtain the pressure $\hat{p}^{k+1}$ from the artificial compressibility equation.

$$
\beta \frac{\partial \hat{p}}{\partial \tau}=-\left(\frac{\partial \hat{u}}{\partial x}+\frac{d \eta}{d y} \frac{\partial \hat{v}}{\partial \eta}\right)
$$

Step 6. Repeat steps 1-5 until the pseudotime problem reaches a steady state.

For each sweep, the unknowns form a tridiagonal matrix. The discretized equations at cell center $i, j$ for the $v$, first $\rho, u$, and second $\rho$ equations, respectively, are

$$
\begin{aligned}
& a_{1 v} \hat{v}_{i j-1}^{k+1}+a_{2 v} \hat{v}_{i j}^{k+1}+a_{3 v} \hat{v}_{i j+1}^{k+1}=a_{4 v}, \\
& a_{1 r 1} \hat{\rho}_{i j-1}^{k+1 / 2}+a_{2 r 1} \hat{\rho}_{i j}^{k+1 / 2}+a_{3 r 1} \hat{\rho}_{i j+1}^{k+1 / 2}=a_{4 r 1}, \\
& a_{1 u} \hat{u}_{i j}^{k+1}+a_{2 u} \hat{u}_{i j}^{k+1}+a_{3 u} \hat{u}_{i \dagger 1 j}^{k+1}=a_{4 u}, \\
& a_{1 r 2} \hat{\rho}_{i-1 j}^{k+1}+a_{2 r 2} \hat{\rho}_{i j}^{k+1}+a_{3 r 2} \hat{\rho}_{i+1 j}^{k+1}=a_{4 r 2},
\end{aligned}
$$

where

$$
\begin{aligned}
a_{1 v}= & \alpha \Delta \tau\left\{-\eta_{v j}^{\prime}\left(v v_{v i j}^{-}-V\right)+\frac{2}{\hat{\rho}_{v i j}^{k}}\left[-\frac{\Delta \tau \eta_{v j}^{\prime} \eta_{u j}^{\prime}}{\beta}\right.\right. \\
& \left.\left.-\frac{1}{\operatorname{Re}}\left(\eta_{v j}^{\prime 2}-\frac{\eta_{2 v j}^{\prime \prime}}{2}\right)\right]\right\} \\
a_{2 v}= & 1+\Delta \tau+\alpha \Delta \tau \eta_{v j}^{\prime} D_{y} v_{i j}+\frac{2 \alpha \Delta \tau}{\hat{\rho}_{v i j}^{k}}\left(\frac { \Delta \tau } { \beta } \eta _ { v j } ^ { \prime } \left(\eta_{u j+1}^{\prime}\right.\right. \\
& \left.\left.+\eta_{u j}^{\prime}\right)+\frac{1}{\operatorname{Re}} 2 \eta_{v j}^{\prime 2}\right), \\
a_{3 v}= & \alpha \Delta \tau\left\{\eta_{v j}^{\prime}\left(v v_{v i j}^{+}-V\right)+\frac{2}{\hat{\rho}_{v i j}^{k}}\left[-\frac{\Delta \tau \eta_{v j}^{\prime} \eta_{u j+1}^{\prime}}{\beta}\right.\right. \\
& \left.\left.-\frac{1}{\operatorname{Re}}\left(\eta_{v j}^{\prime 2}+\frac{\eta_{2 v j}^{\prime \prime}}{2}\right)\right]\right\} \\
a_{4 v}= & \hat{v}_{i j}^{k}-\frac{2 \alpha \Delta \tau \eta_{v j}^{\prime}}{\hat{\rho}_{v i j}^{k}}\left(\hat{\rho}_{i j+1}^{k}-\hat{p}_{i j}^{k}-\frac{\Delta \tau}{\beta} \frac{1}{h}\left(\hat{u}_{i j+1}^{k}\right.\right. \\
& \left.-\hat{u}_{i-1 j+1}^{k}-\hat{u}_{i j}^{k}+\hat{u}_{i-1 j}^{k}\right)+\Delta \tau H_{v i j} \\
\hat{\rho}_{v i j}^{k}= & \hat{\rho}_{i j+1}^{k}+\hat{\rho}_{i j}^{k}+\rho_{i j+1}+\rho_{i j}, \\
a_{1 r 1}= & -\frac{\alpha \Delta \tau}{4}\left(\hat{v}^{k+1}+v-V\right)_{i j P} \eta_{u j p}^{\prime} \\
a_{3 r 1}= & \frac{\alpha \Delta \tau}{4}\left(\hat{v}^{k+1}+v-V\right)_{i j p} \eta_{u j p}^{\prime} \\
& \hat{\rho}_{i j}^{k}+\frac{\Delta \tau}{2} H_{r l i j}, \\
& \Delta \tau
\end{aligned}
$$



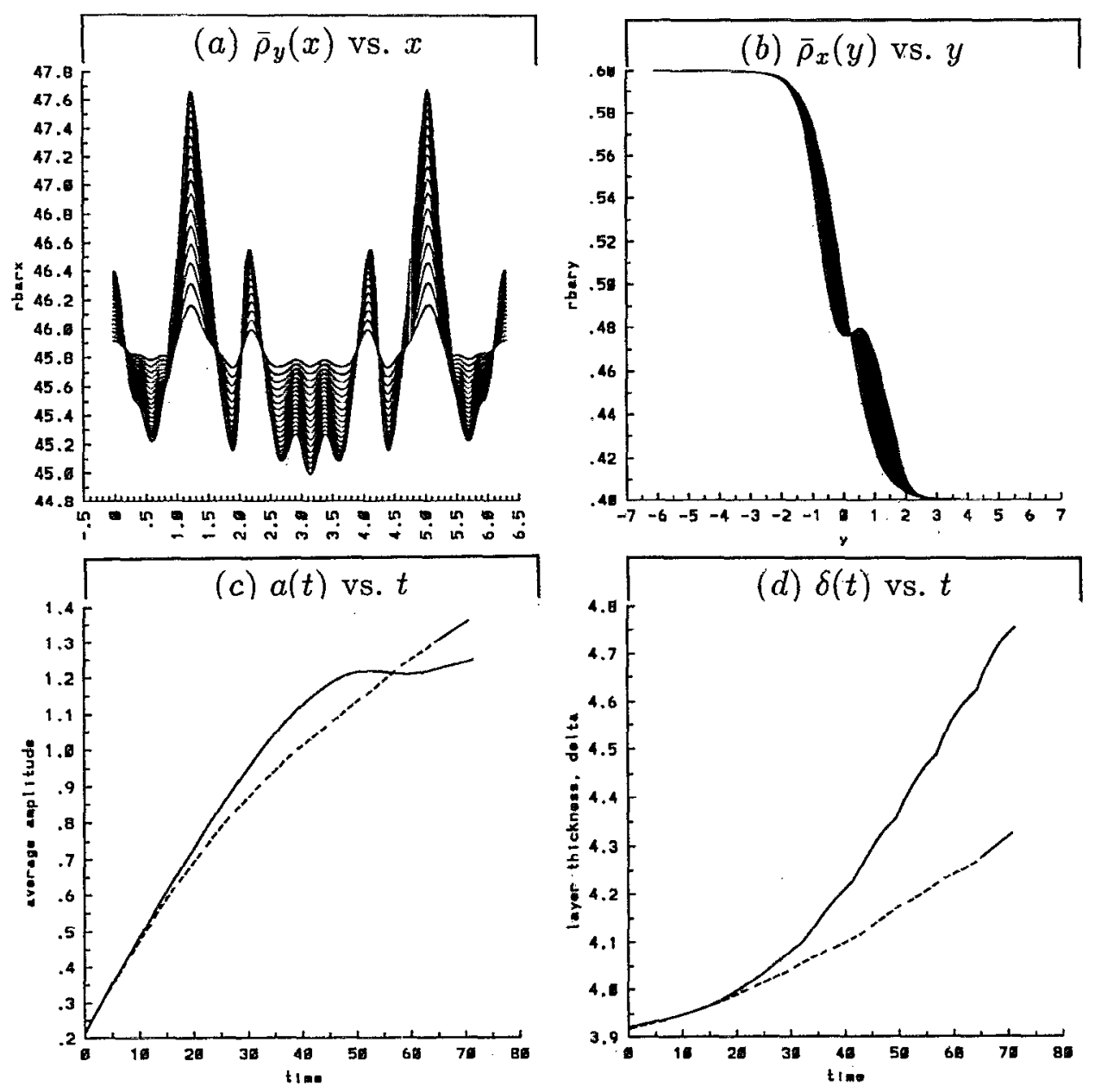

FIG. 25. Time evolution of the average quantities for the first initial random profile, $A=-0.2, t=0$ to $70.5:$ (a) $\bar{\rho}_{y}(x)$; (b) $\bar{\rho}_{x}(y)$. Combined results: -. numerical simulation; ---, linear model; (c) average amplitude; and (d) width of the density laycr.

$$
\begin{aligned}
a_{1 u}= & -\frac{\alpha \Delta \tau}{h} u u_{u i j}^{-}+\frac{2 \alpha \Delta \tau}{\hat{\rho}_{u i j}^{k+1 / 2}}\left(-\frac{\Delta \tau}{\beta h^{2}}-\frac{1}{\operatorname{Re} h^{2}}\right), \\
a_{2 u}= & 1+\Delta \tau+\frac{\alpha \Delta \tau}{h}\left(\frac{u_{i+1 j}-u_{i-1 j}}{2}\right) \\
& +\frac{2 \alpha \Delta \tau}{\hat{\rho}_{u i j}^{k+1 / 2}}\left(\frac{2 \Delta \tau}{\beta h^{2}}+\frac{2}{\operatorname{Re} h^{2}}\right), \\
a_{3 u}= & \frac{\alpha \Delta \tau}{h} u u_{u i j}^{+}+\frac{2 \alpha \Delta \tau}{\hat{\rho}_{u i j}^{k+1 / 2}}\left(-\frac{\Delta \tau}{\beta h^{2}}-\frac{1}{\operatorname{Re} h^{2}}\right), \\
a_{4 u}= & \hat{u}_{i j}^{k}-\frac{\alpha \Delta \tau}{\hat{\rho}_{u i j}^{k+1 / 2}} \frac{1}{h}\left(\hat{p}_{i+1 j}^{k}-\hat{p}_{i j}^{k}+\frac{\Delta \tau}{\beta} \eta_{u j}^{\prime}\left(\hat{v}_{i+1 j-1}^{k+1}\right.\right. \\
& \left.\left.-\hat{v}_{i+1 j}^{k+1}+\hat{v}_{i j}^{k+1}-\hat{v}_{i j-1}^{k+1}\right)\right)+\Delta \tau H_{u i j}, \\
\hat{\rho}_{u i j}^{k+1 / 2}= & \hat{\rho}_{i j}^{k+1 / 2}+\hat{\rho}_{i+1 j}^{k+1 / 2}+\rho_{i j}+\rho_{i+1 j}, \\
a_{1 / 2}= & -\frac{\alpha \Delta \tau}{4 h}\left(\hat{u}^{k+1}+u\right)_{i j P}, \\
a_{2 r 2}= & 1+\frac{\Delta \tau}{2},
\end{aligned}
$$

$$
\begin{aligned}
& a_{3 r 2}=\frac{\alpha \Delta \tau}{4 h}\left(\hat{u}^{k+1}+u\right)_{i j P}, \\
& a_{4 r 2}=\hat{\rho}_{i j}^{k+1 / 2}+\frac{\Delta \tau}{2} H_{r 2 i j} .
\end{aligned}
$$

Dirichlet boundary conditions are enforced during the sweep in the $y$ direction while periodic boundary conditions are enforced during the sweep in the $x$ direction. The iterative scheme of the pseudotime problem has two parameters $\Delta \tau$ and $\beta$ that affect the convergence rate. It should also be noted that other permutations for the sweeping order are possible. However, tests of this method indicate that the order of sweeps does not materially affect the convergence of the scheme. It is shown by $\mathrm{Pham}^{13}$ that the proper choices of $\beta$ and $\Delta \tau$ are strongly problem dependent and will depend in general on the grid size and flow velocities.

${ }^{1}$ R. D. Richtmyer, "Taylor instability in shock acceleration of compressible fiuids," Commun. Pure Appl. Math. 8, 297 (1960).

${ }^{2}$ P. G. Saffman and D. I. Meiron, "Kinetic energy generated by the incompressible Richtmyer-Meshkov instability in a continuously stratified fluid," Phys. Fluids A 1, 1767 (1989).

${ }^{3}$ R. LeLevier, G. J. Lasher, and F. Bjorklund, "Effect of a density gra- 
dient on Taylor instability," Report No. UCRL-4459, University of California Radiation Laboratory, 1955.

${ }^{4}$ R. E. Duff, F. H. Harlow, and C. W. Hirt, "Effects of diffusion on interface instability between gases," Phys. Fluids 5, 417 (1962).

${ }^{5}$ K. O. Mikaelian, "Richtmyer-Meshkov instabilities in stratified fluids," Phys. Rev. A 31, 410 (1985).

${ }^{6} \mathrm{~K}$. O. Mikaelian, "Density gradient stabilization of the RichtmyerMeshkov instability," Phys. Fluids A 3, 2638 (1991).

${ }^{7}$ K. O. Mikaelian, "Kinetic energy of Rayleigh-Taylor and RichtmyerMeshkov instabilities," Phys. Fluids A 3, 2625 (1991).

${ }^{8}$ E. E. Meshkov, "Instability of the interface of two gases accelerated by a shock wave," Sov. Fluid Dyn. 4, 101 (1969).

${ }^{9} \mathrm{M}$. Brouillette, "On the interaction of shock waves with contact surfaces between gases of different densities," Ph.D. thesis, California Institute of Technology, 1989.

${ }^{10} \mathrm{D}$. L. Youngs, "Numerical simulation of turbulent mixing by RayleighTaylor instability," Physica D 12, 32 (1984).

${ }^{11} \mathrm{~K}$. I. Read, "Experimental investigation of turbulent mixing by Rayleigh-Taylor instability," Physica D 12, 45 (1984)

${ }^{12}$ G. I. Barenblatt, "Self-similar turbulence propagation from an instantaneous plane source,"-in Non-Linear Dynamics and Turbulence, edited by G. I. Barenblatt, G. Ioos, and D. D. Joseph (Pitman, Boston, 1983), p. 48 .
${ }^{13}$ T. Pham, "Numerical studies of incompressible Richtmyer-Meshkov instability in a stratified fluid," $\mathrm{Ph}$.D. thesis, California Institute of Technology, 1990.

${ }^{14}$ W. Y. Soh and J. Goodrich, "Unsteady solution of incompressible Navier-Stokes equations," J. Comput. Phys. 79, 113 (1988).

${ }^{15}$ A. J. Chorin, "Numerical solution of the Navier-Stokes equations," Math. Comp. 22, 745 (1968).

${ }^{16}$ H. Lámb, Hydrodynamics (Dover, New York, 1932).

${ }^{17} \mathrm{~F}$. H. Harlow and J. E. Welch, "Numerical calculation of timedependent viscous incompressible flow of fluid with free surface," Phys. Fluids 8, 2182 (1965).

${ }^{18} \mathrm{~K}$. O. Mikaelian, "Simulation of the Richtmyer-Meshkov instability and turbulent mixing in shock-tube experiments," UC Report No. UCID-21328, Lawrence Livermore National Laboratory, Livermore, California, 1988 (unpublished).

${ }^{19}$ J. Glimm, J. W. Grove, Y. Chen, and X. L. Li, "Chaotic mixing at unstable interfaces," in Proceedings of the Third International Workshop on the Physics of Compressible Turbulent Mixing, Royaumont, France, 1991 (CEA, Paris, 1991), p. 19.

${ }^{20}$ D. Youngs, in Ref. 19 , p. 83.

${ }^{21} \mathrm{~J}$. A. Zufiria, "Bubble competition in Rayleigh-Taylor instability," Phys. Fluids 31, 440 (1988). 